\title{
Multiparticle Production and Initial Quasitemperature from Proton-Induced Carbon Collisions at $p_{\mathrm{Lab}}=31 \mathrm{GeV} / \mathrm{c}$
}

\author{
Pei-Pin Yang, ${ }^{1}$ Mai-Ying Duan $\mathbb{D},{ }^{1}$ Fu-Hu Liu $\mathbb{D},{ }^{1}$ and Raghunath Sahoo $\mathbb{D}^{2}$ \\ ${ }^{1}$ Institute of Theoretical Physics and Department of Physics and State Key Laboratory of Quantum Optics and Quantum \\ Optics Devices, Shanxi University, Taiyuan, Shanxi 030006, China \\ ${ }^{2}$ Discipline of Physics, School of Basic Sciences, Indian Institute of Technology Indore, Simrol, Indore 453552, India
}

Correspondence should be addressed to Fu-Hu Liu; fuhuliu@163.com

Received 29 August 2019; Revised 7 November 2019; Accepted 3 December 2019; Published 17 January 2020

Academic Editor: Roelof Bijker

Copyright (c) 2020 Pei-Pin Yang et al. This is an open access article distributed under the Creative Commons Attribution License, which permits unrestricted use, distribution, and reproduction in any medium, provided the original work is properly cited. The publication of this article was funded by $\mathrm{SCOAP}^{3}$.

\begin{abstract}
The momentum spectra of charged pions $\left(\pi^{+}\right.$and $\left.\pi^{-}\right)$and kaons $\left(K^{+}\right.$and $\left.K^{-}\right)$, as well as protons $(p)$, produced in the beam proton-induced collisions in a $90 \mathrm{~cm}$ long graphite target (proton-carbon $(p-\mathrm{C})$ collisions) at the beam momentum $p_{\text {Lab }}=31$ $\mathrm{GeV} / \mathrm{c}$ are studied in the framework of a multisource thermal model by using Boltzmann distribution and the Monte Carlo method. The theoretical model results are approximately in agreement with the experimental data measured by the NA61/SHINE Collaboration. The related free parameters (effective temperature, rapidity shifts, and fraction of nonleading protons) and derived quantities (average transverse momentum and initial quasitemperature) under given experimental conditions are obtained. The considered free parameters and derived quantities are shown to be strongly dependent on the emission angle over a range from 0 to $380 \mathrm{mrad}$ and weakly dependent on longitudinal position (graphite target thickness) over a range from 0 to $90 \mathrm{~cm}$.
\end{abstract}

\section{Introduction}

High-energy (relativistic) nucleus-nucleus (heavy ion) collisions with nearly zero impact parameter (central collisions) are believed to form Quark-Gluon Plasma (QGP) or quark matter $[1-3]$ in the laboratory. High-energy nucleusnucleus collisions with a large impact parameter are not expected to form QGP due to low particle multiplicity yielding lower energy density and temperature [4]. Small collision systems such as proton-nucleus and proton-proton collisions at high energy produce usually low multiplicity, which are not expected to form QGP but are useful to study the multiparticle production processes. However, a few of protonnucleus and proton-proton collisions at the LHC energies can produce high multiplicity due to the nearly zero "impact parameter," which are possibly expected to form QGP, where the concept "impact parameter" or "centrality" used in nuclear collisions are used in proton-proton collisions [5]. Degree of collectivity, long-range correlations, strangeness enhancement, etc., which are considered as QGP-like signatures, are recently observed in these high multiplicity events [6-8].

Assuming nucleus-nucleus collisions as a mere superposition of proton-proton collisions in the absence of any nuclear effects, usually one considers proton-proton collisions as the baseline measurements. On the other hand, proton-nucleus collisions [9-13] serve to study the initial state effects and make a bridge between proton-proton [14-18] to nucleus-nucleus collisions [19-23] while studying the multiparticle production processes, though fewer particles are produced in proton-nucleus collisions than in nucleus-nucleus collisions.

There are different types of models or theories being introduced in the studies of high-energy collisions [24, 25]. Among these models or theories, different versions of thermal and statistical models [26-29] characterize some of the aspects of high-energy nuclear collisions, while there are many other aspects that are studied by other approaches. 

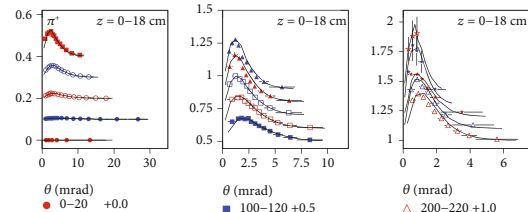

$\begin{aligned} & \theta \text { (mrad) } \\ & -0-20\end{aligned}+0.0$

- $20-40+0.1$

$40-60+0.22$

- $80-100+0.4$

(a)

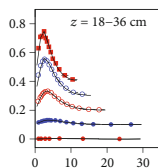

$\theta$ (mrad) $0-20+0.0$

- $20-40+0.1$

- $40-60+0.2$

$60-80+0.3$
- $80-100+0.4$

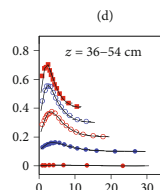

$\theta$ (mrad)

- $20-40+0.1$

$\circ 40-60+0.2$

60-80+0.3

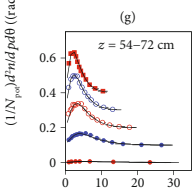

\begin{tabular}{llll}
\hline 0 & 10 & 20 & 30 \\
\hline
\end{tabular}

$\begin{aligned} & \theta \text { (mrad) } \\ & -0-20\end{aligned}+0.0$

- $20-40+0.1$

$-40-60+0.2$
$0.60-80+03$

- $80-100+0.4$

(10)

$\begin{aligned} & \theta \text { (mrad) } \\ & 0-20\end{aligned}+0.0$

- 20-40+0.1

○ $40-60+0.2$

○ $60-80+0.3$

$80-100+0.4$

(m)

- $100-120+0.5$

ㅁ $140-160+0.7$

$\triangle 160-180+0.8$

$180-200+0.9$

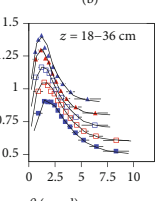

$\theta$ (mrad)
- $100-120+0.5$
$\square$

ㅁ $120-140+0.6$

$140-160+0.7$

$180-200+0.9$

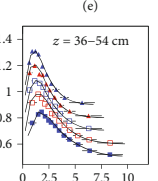

$\theta$ (mrad)

$\triangle 220-260+1.1$

$\star 260-300+1.2$

* $300-340+1.3$
$* \quad 340-380+1.4$

(c)

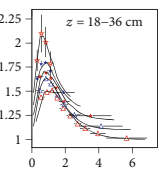

$\theta(\mathrm{mrad})$
$\triangle 200-220+$

$\triangle 220-260+1.1$

$\star 260-300+1.2$

* $300-340+1.3$

* $300-340+1.3$

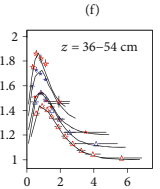

$\theta(\mathrm{mrad}))$

- $100-120+0.5$

ㅁ $120-140+0.6$

- $160-180+08$

-160-180 +0.8

(h)

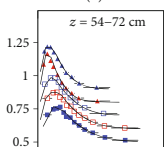

$\theta(\mathrm{mrad})$
$\triangle 200-220+1.0$

- 220-260+1.1

$\Delta 20-260+1.1$
$* \quad 260-300+1.2$

× $300-340+1.3$

* $300-340+1.3$

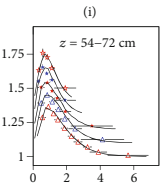

$\theta$ (mrad)
$100-120+0.5$

- $120-140+0.6$

ㅁ $140-160+0.7$

160-180 +0.

A $180-200+0.9$

(k)

$\triangle 220-260+1.1$

* $260-300+1.2$

* $300-340+1.3$
$* \quad 340-380+1.4$
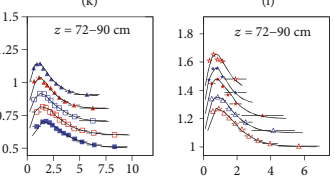

$\theta(\mathrm{mrad})$
$100-120+0.5$

- $120-140+0.6$

140-160 +0.

A $160-180+0.8$

- 180-200+0.9

(n)

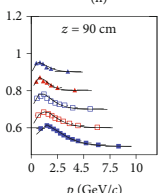

$\begin{aligned} & \theta(\mathrm{mrad}) \\ & 0-20\end{aligned}+0.0$

- 20-40 +0.1

○ 40-60 +0

ㅇ $60-80+0.3$

- $80-100+0.4$
$(\mathrm{p})$

$\theta$ (mrad)

- $100-140+0.5$

1 140-180+0.6

180-220 +0.7

$220-260+0.8$

A $260-300+0$.

Figure 1: Momentum spectra of $\pi^{+}$produced in $p$-C collisions at $31 \mathrm{GeV} / c$. Panels $(\mathrm{a}-\mathrm{c}),(\mathrm{d}-\mathrm{f}),(\mathrm{g}-\mathrm{i}),(\mathrm{j}-\mathrm{l}),(\mathrm{m}-\mathrm{o})$, and $(\mathrm{p}-\mathrm{q})$ represent the spectra for $z=0-18,18-36,36-54,54-72,72-90$, and $90 \mathrm{~cm}$, respectively. The symbols represent the experimental data [32]. The curves are our results fitted by the multisource thermal model due to Equation (1) and the Monte Carlo method. To show clearly, different spectra are scaled by adding different amounts marked in the panels.

$\theta(\mathrm{mrad})$
$\Delta 200-220+1.0$

$\Delta 20-260+1.1$
$\star 260-300+1.2$

$\star 300-340+1.3$

(o)
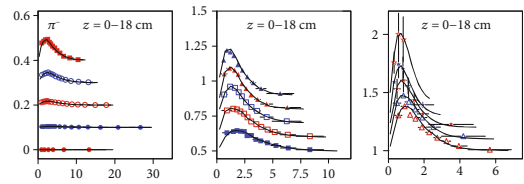

$\begin{array}{lll}\theta(\mathrm{mrad}) & \theta(\mathrm{mrad}) & \theta(\mathrm{mrad}) \\ 0-20 \quad+0.0 & \quad 100-120+0.5 & \Delta 200-220+1.0\end{array}$

- $20-40+0.1 \quad \square 120-140+0.6 \quad \Delta 220-260+1.1$

$\begin{array}{lll}\circ & 40-60+0.2 \quad \square 140-160+0.7 & \text { * } 260-300+1.2\end{array}$

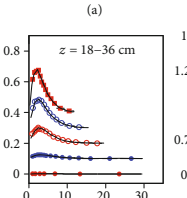

(b)

* $340-380+1.4$
(c)

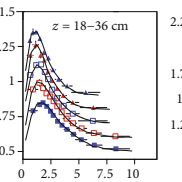

$\theta(\bmod )-250$

$\theta(\mathrm{mrad})$
- $100-120+0.5$
$\square$

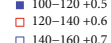

$\triangle 160-180+0.8$

$\Delta 180-200+0.9$

$00-80+03.30$

$060-80+0.3$

(d)
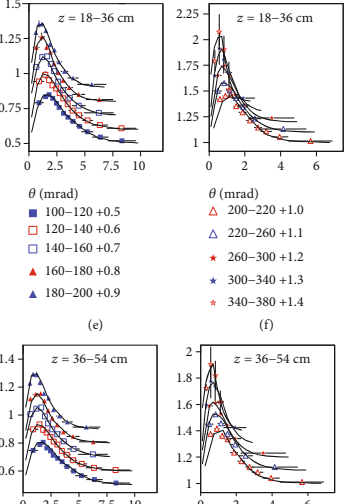

$\theta(\mathrm{mrad})$
$\Delta 200-220+1.0$

$\Delta 220-260+1+1$

$\Delta 20-260+1.1$
$\star 260-300+1.2$

$\star 260-300+1.2$

* $300-340+1.3$

(I)

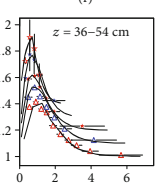

$\theta$ (mrad)
$0-20 \quad+0.0$

$\begin{array}{lll}0-20-40 & +0.0 \\ 0.1 & \end{array}$

$\theta$ (mrad)

$\circ$ ) $40-60+0.2$
$\circ 60-80+0.3$

- $100-120+0.5$

口 140-160+0.7

A $160-180+0.8$

- $80-100+0.4$

救

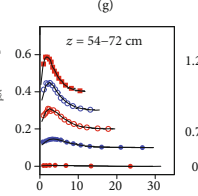

$\theta$ (mrad)
$\Delta \quad 200-220+1.0$

$\Delta 220-260+1.1$

$260-300+1.2$
$300-340+1.3$

* 340-380 +1.4

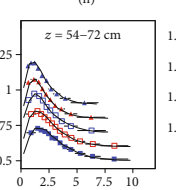

$\begin{aligned} & \theta(\mathrm{mrad}) \\ & -0-20\end{aligned}+0.0$

$\begin{array}{ll}-0-20 & +0.0 \\ -20-40 & +0.1\end{array}$

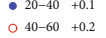

o $60-80+0.3$

- $80-100+0.4$

(j)

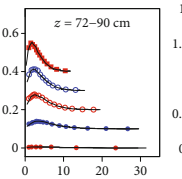

$\theta$ (mrad)

- $100-120+0.5$

$\square 120-140+0.6$
$\square \quad 140-160+0.7$
$4160-180+0.8$

1 $160-180+0.8$

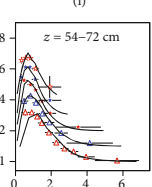

$\theta$ (mrad)

$\Delta \quad 200-220+1.0$
$\triangle \quad 220-260+1.1$

$\triangle 220-260+1.1$

* $260-300+1.2$

* $300-340+1.3$

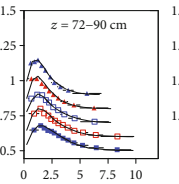

(1)

$\theta$ (mrad)

- $0-20 \quad+0.0$

\begin{tabular}{lll}
$-20-40$ & +0.1 \\
\hline & $40-60$ & +0.2
\end{tabular}

○ $40-60+0.2$

을 $60-80+0.3$
- $80-100+0.4$

- $100-120+0.5$

ㅁ $120-140+160+0.6$

A $160-180+0.8$

\begin{tabular}{l}
$\Delta 180-200+0.9$ \\
\hline
\end{tabular}

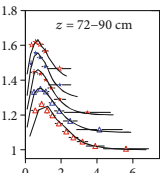

$\theta$ (mrad)

$\triangle 220-260+1.1$

$\Delta 220-260+1.1$
$\times \quad 260-300+1.2$

$\star 260-300+1.2$
$\star$
$* 300-340+1.3$

$\star 300-340+1.3$
$\star \quad 340-380+1.4$
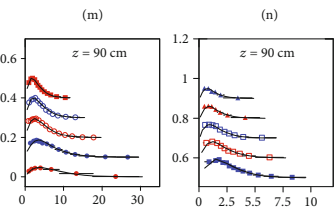

(o)

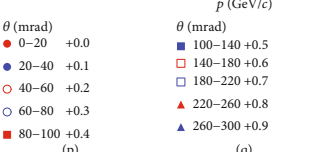

Figure 2: Same as Figure 1 but showing the spectra of $\pi^{-}$.

As a basic concept, temperature is ineluctable to be used in analyses. In fact, not only is it that "temperature is surely one of the central concepts in thermodynamics and statistical mechanics" [30], but it is also very important due to its 
TABLE 1: Values of $T, y_{\max }, y_{\min }, N_{0}, \chi^{2}$, and ndof corresponding to the curves in Figure 1 in which different data are measured in different $\theta$ and $z$ ranges. In the table, $z$ is in the units of $\mathrm{cm}$ and $\theta$ is not listed, which appears in Figure 1. In one case, ndof is negative which appears in terms of "-" and the corresponding curve is just for eye guiding purpose.

\begin{tabular}{|c|c|c|c|c|c|}
\hline Figure & $T(\mathrm{GeV})$ & $y_{\max }$ & $y_{\min }$ & $N_{0}(\times 0.001)$ & $\chi^{2} /$ ndof \\
\hline \multirow{5}{*}{$\begin{array}{l}\text { Figure } 1 \text { (a) } \\
0<z<18\end{array}$} & $0.320 \pm 0.005$ & $2.30 \pm 0.02$ & $1.10 \pm 0.02$ & $0.143 \pm 0.010$ & $22 / 2$ \\
\hline & $0.138 \pm 0.004$ & $3.25 \pm 0.05$ & $1.72 \pm 0.06$ & $0.962 \pm 0.020$ & $42 / 12$ \\
\hline & $0.195 \pm 0.006$ & $2.47 \pm 0.04$ & $1.57 \pm 0.03$ & $2.918 \pm 0.100$ & $93 / 12$ \\
\hline & $0.205 \pm 0.003$ & $2.36 \pm 0.04$ & $1.47 \pm 0.03$ & $6.431 \pm 0.200$ & $87 / 9$ \\
\hline & $0.220 \pm 0.005$ & $2.09 \pm 0.04$ & $1.00 \pm 0.03$ & $11.108 \pm 0.400$ & $85 / 9$ \\
\hline \multirow{5}{*}{$\begin{array}{l}\text { Figure } 1 \text { (b) } \\
0<z<18\end{array}$} & $0.222 \pm 0.004$ & $2.00 \pm 0.03$ & $0.80 \pm 0.04$ & $14.541 \pm 0.300$ & $60 / 9$ \\
\hline & $0.169 \pm 0.003$ & $2.20 \pm 0.04$ & $0.78 \pm 0.02$ & $16.701 \pm 0.340$ & $15 / 9$ \\
\hline & $0.166 \pm 0.003$ & $2.10 \pm 0.03$ & $0.45 \pm 0.02$ & $17.572 \pm 0.260$ & $16 / 6$ \\
\hline & $0.166 \pm 0.002$ & $2.00 \pm 0.03$ & $0.65 \pm 0.03$ & $19.149 \pm 0.300$ & $37 / 6$ \\
\hline & $0.166 \pm 0.002$ & $1.90 \pm 0.02$ & $0.65 \pm 0.02$ & $18.495 \pm 0.340$ & $57 / 6$ \\
\hline \multirow{5}{*}{$\begin{array}{l}\text { Figure } 1(\mathrm{c}) \\
0<z<18\end{array}$} & $0.166 \pm 0.001$ & $1.80 \pm 0.02$ & $0.65 \pm 0.02$ & $17.839 \pm 0.200$ & $70 / 6$ \\
\hline & $0.136 \pm 0.002$ & $2.08 \pm 0.06$ & $0.75 \pm 0.04$ & $31.163 \pm 0.720$ & $35 / 3$ \\
\hline & $0.160 \pm 0.003$ & $1.75 \pm 0.03$ & $0.55 \pm 0.02$ & $25.050 \pm 0.560$ & $28 / 2$ \\
\hline & $0.115 \pm 0.004$ & $2.08 \pm 0.08$ & $0.90 \pm 0.05$ & $26.483 \pm 0.800$ & $12 / 1$ \\
\hline & $0.115 \pm 0.004$ & $2.08 \pm 0.04$ & $0.85 \pm 0.10$ & $25.278 \pm 1.200$ & $2 /-$ \\
\hline \multirow{5}{*}{$\begin{array}{l}\text { Figure } 1 \text { (d) } \\
18<z<36\end{array}$} & $0.320 \pm 0.010$ & $2.50 \pm 0.02$ & $1.48 \pm 0.01$ & $0.571 \pm 0.020$ & $49 / 3$ \\
\hline & $0.210 \pm 0.004$ & $2.73 \pm 0.02$ & $1.50 \pm 0.04$ & $5.224 \pm 0.080$ & $48 / 12$ \\
\hline & $0.198 \pm 0.003$ & $2.56 \pm 0.04$ & $1.57 \pm 0.03$ & $17.336 \pm 0.400$ & $76 / 12$ \\
\hline & $0.215 \pm 0.004$ & $2.30 \pm 0.03$ & $1.35 \pm 0.02$ & $28.156 \pm 1.000$ & $90 / 9$ \\
\hline & $0.224 \pm 0.004$ & $2.09 \pm 0.03$ & $1.00 \pm 0.02$ & $31.294 \pm 0.400$ & $67 / 9$ \\
\hline \multirow{5}{*}{$\begin{array}{l}\text { Figure } 1(\mathrm{e}) \\
18<z<36\end{array}$} & $0.222 \pm 0.005$ & $1.90 \pm 0.04$ & $0.70 \pm 0.03$ & $31.345 \pm 0.800$ & $70 / 9$ \\
\hline & $0.169 \pm 0.004$ & $2.20 \pm 0.03$ & $0.80 \pm 0.05$ & $30.855 \pm 0.400$ & $29 / 9$ \\
\hline & $0.172 \pm 0.005$ & $2.10 \pm 0.02$ & $0.45 \pm 0.02$ & $28.588 \pm 0.600$ & $3 / 6$ \\
\hline & $0.168 \pm 0.002$ & $1.90 \pm 0.04$ & $0.55 \pm 0.05$ & $25.800 \pm 0.400$ & $19 / 6$ \\
\hline & $0.166 \pm 0.001$ & $1.90 \pm 0.02$ & $0.65 \pm 0.01$ & $24.237 \pm 0.200$ & $50 / 6$ \\
\hline \multirow{5}{*}{$\begin{array}{l}\text { Figure } 1 \text { (f) } \\
18<z<36\end{array}$} & $0.167 \pm 0.002$ & $1.78 \pm 0.03$ & $0.65 \pm 0.02$ & $22.902 \pm 0.400$ & $64 / 6$ \\
\hline & $0.137 \pm 0.001$ & $1.95 \pm 0.02$ & $0.75 \pm 0.02$ & $38.121 \pm 0.800$ & $44 / 3$ \\
\hline & $0.160 \pm 0.002$ & $1.75 \pm 0.03$ & $0.45 \pm 0.03$ & $33.929 \pm 0.800$ & $22 / 2$ \\
\hline & $0.155 \pm 0.002$ & $1.70 \pm 0.03$ & $0.45 \pm 0.02$ & $30.541 \pm 0.800$ & $6 / 1$ \\
\hline & $0.182 \pm 0.003$ & $0.70 \pm 0.02$ & $0.55 \pm 0.03$ & $31.012 \pm 0.600$ & $7 / 0$ \\
\hline \multirow{5}{*}{$\begin{array}{l}\text { Figure } 1 \text { (g) } \\
36<z<54\end{array}$} & $0.300 \pm 0.002$ & $2.50 \pm 0.02$ & $1.40 \pm 0.02$ & $1.064 \pm 0.030$ & $38 / 3$ \\
\hline & $0.193 \pm 0.003$ & $2.85 \pm 0.01$ & $1.55 \pm 0.02$ & $10.836 \pm 0.200$ & $108 / 12$ \\
\hline & $0.195 \pm 0.003$ & $2.60 \pm 0.05$ & $1.57 \pm 0.03$ & $23.318 \pm 0.400$ & $85 / 12$ \\
\hline & $0.215 \pm 0.005$ & $2.30 \pm 0.03$ & $0.95 \pm 0.03$ & $21.566 \pm 0.600$ & $60 / 9$ \\
\hline & $0.220 \pm 0.003$ & $2.09 \pm 0.02$ & $0.90 \pm 0.02$ & $27.103 \pm 0.400$ & $69 / 9$ \\
\hline \multirow{5}{*}{$\begin{array}{l}\text { Figure } 1 \text { (h) } \\
36<z<54\end{array}$} & $0.242 \pm 0.002$ & $1.88 \pm 0.02$ & $0.25 \pm 0.03$ & $26.968 \pm 0.600$ & $42 / 9$ \\
\hline & $0.169 \pm 0.003$ & $2.20 \pm 0.02$ & $0.70 \pm 0.03$ & $26.177 \pm 0.400$ & $29 / 9$ \\
\hline & $0.168 \pm 0.003$ & $2.10 \pm 0.04$ & $0.40 \pm 0.05$ & $22.962 \pm 0.400$ & $13 / 6$ \\
\hline & $0.166 \pm 0.005$ & $1.95 \pm 0.03$ & $0.30 \pm 0.02$ & $21.297 \pm 0.600$ & $40 / 6$ \\
\hline & $0.166 \pm 0.002$ & $1.93 \pm 0.03$ & $0.35 \pm 0.04$ & $19.830 \pm 0.500$ & $44 / 6$ \\
\hline
\end{tabular}


TABle 1: Continued.

\begin{tabular}{|c|c|c|c|c|c|}
\hline Figure & $T(\mathrm{GeV})$ & $y_{\max }$ & $y_{\min }$ & $N_{0}(\times 0.001)$ & $\chi^{2} /$ ndof \\
\hline \multirow{5}{*}{$\begin{array}{l}\text { Figure } 1(\mathrm{i}) \\
36<z<54\end{array}$} & $0.166 \pm 0.002$ & $1.70 \pm 0.07$ & $0.25 \pm 0.05$ & $17.778 \pm 0.300$ & $97 / 6$ \\
\hline & $0.138 \pm 0.002$ & $1.95 \pm 0.03$ & $0.75 \pm 0.04$ & $32.081 \pm 0.800$ & $37 / 3$ \\
\hline & $0.160 \pm 0.003$ & $1.75 \pm 0.05$ & $0.20 \pm 0.04$ & $23.055 \pm 0.600$ & $5 / 2$ \\
\hline & $0.155 \pm 0.005$ & $1.70 \pm 0.05$ & $0.35 \pm 0.04$ & $26.196 \pm 0.800$ & $6 / 1$ \\
\hline & $0.186 \pm 0.005$ & $0.70 \pm 0.07$ & $0.50 \pm 0.05$ & $21.033 \pm 0.600$ & $22 / 1$ \\
\hline \multirow{5}{*}{$\begin{array}{l}\text { Figure } 1(\mathrm{j}) \\
54<\cdot z<72\end{array}$} & $0.300 \pm 0.004$ & $2.70 \pm 0.02$ & $0.80 \pm 0.04$ & $1.561 \pm 0.040$ & $66 / 3$ \\
\hline & $0.188 \pm 0.001$ & $2.93 \pm 0.02$ & $1.60 \pm 0.02$ & $11.850 \pm 0.200$ & $145 / 12$ \\
\hline & $0.195 \pm 0.003$ & $2.60 \pm 0.03$ & $1.50 \pm 0.02$ & $18.466 \pm 0.400$ & $81 / 12$ \\
\hline & $0.215 \pm 0.005$ & $2.30 \pm 0.03$ & $0.80 \pm 0.03$ & $21.028 \pm 0.500$ & $60 / 9$ \\
\hline & $0.220 \pm 0.004$ & $2.10 \pm 0.03$ & $0.80 \pm 0.02$ & $21.284 \pm 0.400$ & $72 / 9$ \\
\hline \multirow{5}{*}{$\begin{array}{l}\text { Figure } 1(\mathrm{k}) \\
54<z<72\end{array}$} & $0.242 \pm 0.005$ & $1.80 \pm 0.02$ & $0.50 \pm 0.03$ & $19.705 \pm 0.300$ & $76 / 9$ \\
\hline & $0.169 \pm 0.002$ & $2.20 \pm 0.02$ & $0.85 \pm 0.03$ & $19.159 \pm 0.400$ & $36 / 9$ \\
\hline & $0.168 \pm 0.004$ & $2.10 \pm 0.04$ & $0.30 \pm 0.03$ & $16.753 \pm 0.400$ & $13 / 6$ \\
\hline & $0.165 \pm 0.001$ & $1.60 \pm 0.01$ & $0.30 \pm 0.01$ & $14.510 \pm 0.200$ & $87 / 6$ \\
\hline & $0.166 \pm 0.001$ & $1.85 \pm 0.03$ & $0.30 \pm 0.02$ & $15.317 \pm 0.300$ & $35 / 6$ \\
\hline \multirow{5}{*}{$\begin{array}{l}\text { Figure } 1(1) \\
54<z<72\end{array}$} & $0.166 \pm 0.001$ & $1.70 \pm 0.05$ & $0.35 \pm 0.03$ & $14.737 \pm 0.200$ & $94 / 6$ \\
\hline & $0.138 \pm 0.002$ & $2.20 \pm 0.02$ & $0.60 \pm 0.02$ & $26.931 \pm 0.400$ & $22 / 3$ \\
\hline & $0.160 \pm 0.002$ & $1.75 \pm 0.03$ & $0.20 \pm 0.03$ & $23.055 \pm 0.400$ & $5 / 2$ \\
\hline & $0.155 \pm 0.002$ & $1.50 \pm 0.04$ & $0.35 \pm 0.03$ & $20.234 \pm 0.240$ & $8 / 1$ \\
\hline & $0.182 \pm 0.001$ & $0.85 \pm 0.02$ & $0.65 \pm 0.01$ & $17.919 \pm 0.280$ & $16 / 1$ \\
\hline \multirow{5}{*}{$\begin{array}{l}\text { Figure } 1(\mathrm{~m}) \\
54<z<72\end{array}$} & $0.320 \pm 0.005$ & $2.60 \pm 0.03$ & $1.00 \pm 0.03$ & $1.791 \pm 0.020$ & $49 / 3$ \\
\hline & $0.210 \pm 0.003$ & $2.80 \pm 0.02$ & $1.60 \pm 0.03$ & $10.081 \pm 0.300$ & $56 / 12$ \\
\hline & $0.195 \pm 0.002$ & $2.65 \pm 0.03$ & $1.30 \pm 0.03$ & $13.984 \pm 0.400$ & $67 / 12$ \\
\hline & $0.215 \pm 0.004$ & $2.30 \pm 0.02$ & $0.70 \pm 0.03$ & $15.619 \pm 0.300$ & $52 / 9$ \\
\hline & $0.220 \pm 0.003$ & $2.10 \pm 0.03$ & $0.50 \pm 0.03$ & $15.793 \pm 0.400$ & $66 / 9$ \\
\hline \multirow{5}{*}{$\begin{array}{l}\text { Figure } 1(\mathrm{n}) \\
72<z<90\end{array}$} & $0.242 \pm 0.003$ & $1.80 \pm 0.02$ & $0.40 \pm 0.02$ & $15.019 \pm 0.300$ & $64 / 9$ \\
\hline & $0.169 \pm 0.003$ & $2.14 \pm 0.02$ & $0.85 \pm 0.03$ & $14.177 \pm 0.400$ & $73 / 9$ \\
\hline & $0.168 \pm 0.004$ & $2.10 \pm 0.03$ & $0.30 \pm 0.03$ & $12.801 \pm 0.300$ & $9 / 6$ \\
\hline & $0.210 \pm 0.003$ & $1.60 \pm 0.03$ & $0.30 \pm 0.02$ & $11.752 \pm 0.200$ & $14 / 6$ \\
\hline & $0.175 \pm 0.003$ & $1.80 \pm 0.05$ & $0.20 \pm 0.03$ & $11.763 \pm 0.200$ & $36 / 6$ \\
\hline \multirow{5}{*}{$\begin{array}{l}\text { Figure } 1 \text { (o) } \\
72<z<90\end{array}$} & $0.166 \pm 0.005$ & $1.70 \pm 0.04$ & $0.25 \pm 0.05$ & $11.174 \pm 0.200$ & $66 / 6$ \\
\hline & $0.138 \pm 0.002$ & $2.00 \pm 0.03$ & $0.50 \pm 0.03$ & $18.580 \pm 0.400$ & $35 / 3$ \\
\hline & $0.160 \pm 0.002$ & $1.75 \pm 0.02$ & $0.20 \pm 0.02$ & $18.836 \pm 0.200$ & $13 / 2$ \\
\hline & $0.155 \pm 0.002$ & $1.50 \pm 0.06$ & $0.35 \pm 0.02$ & $14.886 \pm 0.400$ & $3 / 1$ \\
\hline & $0.200 \pm 0.003$ & $0.85 \pm 0.04$ & $0.45 \pm 0.03$ & $14.089 \pm 0.400$ & $19 / 1$ \\
\hline \multirow{5}{*}{$\begin{array}{l}\text { Figure } 1(\mathrm{p}) \\
z=90\end{array}$} & $0.320 \pm 0.001$ & $2.60 \pm 0.01$ & $1.00 \pm 0.01$ & $16.231 \pm 0.200$ & $46 / 3$ \\
\hline & $0.200 \pm 0.003$ & $2.80 \pm 0.02$ & $1.60 \pm 0.03$ & $20.333 \pm 0.200$ & $97 / 12$ \\
\hline & $0.195 \pm 0.005$ & $2.62 \pm 0.03$ & $1.30 \pm 0.03$ & $16.733 \pm 0.200$ & $88 / 12$ \\
\hline & $0.210 \pm 0.004$ & $2.30 \pm 0.02$ & $0.70 \pm 0.03$ & $13.641 \pm 0.300$ & $50 / 9$ \\
\hline & $0.210 \pm 0.002$ & $2.00 \pm 0.01$ & $0.40 \pm 0.02$ & $10.621 \pm 0.200$ & $104 / 9$ \\
\hline
\end{tabular}


TABLe 1: Continued.

\begin{tabular}{lccccc}
\hline Figure & $T(\mathrm{GeV})$ & $y_{\max }$ & $y_{\min }$ & $N_{0}(\times 0.001)$ & $14.572 \pm 0.400$ \\
\hline & $0.242 \pm 0.003$ & $1.60 \pm 0.02$ & $0.10 \pm 0.02$ & $8.858 \pm 0.400$ & $83 / 9$ \\
Figure 1(q) & $0.169 \pm 0.003$ & $1.80 \pm 0.03$ & $0.90 \pm 0.02$ & $7.038 \pm 0.120$ & $64 / 6$ \\
$z=90$ & $0.170 \pm 0.002$ & $1.60 \pm 0.03$ & $0.60 \pm 0.02$ & $4.561 \pm 0.240$ & $71 / 3$ \\
& $0.240 \pm 0.002$ & $1.00 \pm 0.02$ & $0.20 \pm 0.03$ & $3.306 \pm 0.200$ & $29 / 2$ \\
\hline
\end{tabular}

extremely wide applications in experimental measurements and theoretical studies in subatomic physics, especially in high energy and nuclear physics.

In view of this importance, in this paper, we are interested in the study of proton-nucleus collisions at high energy by using the Boltzmann distribution and the Monte Carlo method in the framework of the multisource thermal model [31]. The theoretical model results are compared with the experimental data of the beam proton-induced collisions in a $90 \mathrm{~cm}$ long graphite target (proton-carbon $(p-\mathrm{C})$ collisions) at the beam momentum $p_{\mathrm{Lab}}=31 \mathrm{GeV} / c$ measured by the NA61/SHINE Collaboration [32] at the Super Proton Synchrotron (SPS), the European Organisation for Nuclear Research or the European Laboratory for Particle Physics (CERN).

The remainder of this paper is structured as follows. The formalism and method are shortly described in Section 2. Results and discussion are given in Section 3. In Section 4, we summarize our main observations and conclusions.

\section{Formalism and Method}

According to the multisource thermal model [31], it is assumed that there are many local emission sources to be formed in high-energy collisions due to different excitation degrees, rapidity shifts, reaction mechanisms, and impact parameters (or centralities). In the transverse plane, the local emission sources with the same excitation degree form a (large) emission source. In the rapidity space, the local emission sources with the same rapidity shift form a (large) emission source. In the rest frame of an emission source with a determined excitation degree, the particles are assumed to be emitted isotropically.

In the rest frame of a given emission source, let $T$ denote the temperature parameter. The particles with rest mass $m_{0}$ produced in the rest frame of the emission source are assumed to have the simplest Boltzmann distribution of momenta $p^{\prime}[33]$. That is,

$$
f_{p^{\prime}}\left(p^{\prime}\right)=C p^{\prime 2} \exp \left(-\frac{\sqrt{p^{\prime 2}+m_{0}^{2}}}{T}\right)
$$

where $C$ is the normalization constant which is related to $T$. As a probability density function, Equation (1) is naturally normalized to 1 .
If we need to consider multiple sources, we can use a superposition of different equations with different temperatures and fractions. We have

$$
f_{p^{\prime}}\left(p^{\prime}\right)=\sum_{j} k_{j} C_{j} p^{\prime 2} \exp \left(-\frac{\sqrt{p^{\prime 2}+m_{0}^{2}}}{T_{j}}\right)
$$

where $k_{j}, C_{j}$, and $T_{j}$ are the fraction, normalization constant, and temperature for the $j$-th source or component, respectively. The average temperature obtained from Equation (2) is $T=\sum_{j} k_{j} T_{j} / \sum_{j} k_{j}=\sum_{j} k_{j} T_{j}$ due to $\sum_{j} k_{j}=1$. The derived parameter $T$ is the weighted average over various components, but not the simple weighted sum.

It should be noted that $T$ or $T_{j}$ is not the "real" temperature of the emission source, but the effective temperature due to the fact that the flow effect is not excluded in the momentum spectrum. The "real" temperature is generally smaller than the effective temperature which contains the contribution of the collective radial flow effect. To disengage the thermal motion and collective flow effect, one may use different methods such as the blast-wave model $[34,35]$ or any alternative method [36, 37]. As an example, we shall discuss shortly the results of the blast-wave model in Section 3.

The contribution of spin being small is not included in Equation (1). The effect of chemical potential $(\mu)$ is not included in Equation (1) as well, due to the fact that $\mu$ affects only the normalization, but not the trend, of the spectrum if the spin effect is neglected. Our previous work [38] shows that the spin effect together with $\mu \gg m_{0}$ or $\mu \ll m_{0}$ is so small $(<1 \%)$ that we do not need to consider it in studying momentum or transverse momentum spectra in highenergy collisions. Only the combination of spin and $\mu \approx m_{0}$ causes an obvious effect, which is not the case in this paper.

In the Monte Carlo method $[39,40]$, let $R_{1,2,3,4}$ denote random numbers distributed evenly in $[0,1]$. To obtain a concrete value of $p^{\prime}$ which satisfies Equation (1) or one of the components in Equation (2), we can perform the solution of

$$
\int_{0}^{p^{\prime}} f_{p^{\prime}}\left(p^{\prime \prime}\right) d p^{\prime \prime}<R_{1}<\int_{0}^{p^{\prime}+\delta p^{\prime}} f_{p^{\prime}}\left(p^{\prime \prime}\right) d p^{\prime \prime},
$$

where $\delta p^{\prime}$ denotes a small shift relative to $p^{\prime}$. 
TABLe 2: Values of $T, y_{\max }, y_{\min }, N_{0}, \chi^{2}$, and ndof corresponding to the curves in Figure 2 in which different data are measured in different $\theta$ and $z$ ranges. In the table, $z$ is in the units of $\mathrm{cm}$ and $\theta$ is not listed, which appears in Figure 2. In one case, ndof is negative which appears in terms of "-" and the corresponding curve is just for eye guiding only.

\begin{tabular}{|c|c|c|c|c|c|}
\hline Figure & $T(\mathrm{GeV})$ & $y_{\max }$ & $y_{\min }$ & $N_{0}(\times 0.001)$ & $\chi^{2} /$ ndof \\
\hline \multirow{5}{*}{$\begin{array}{l}\text { Figure 2(a) } \\
0<z<18\end{array}$} & $0.320 \pm 0.005$ & $2.30 \pm 0.02$ & $1.10 \pm 0.02$ & $0.077 \pm 0.002$ & $33 / 2$ \\
\hline & $0.138 \pm 0.004$ & $3.00 \pm 0.03$ & $1.72 \pm 0.03$ & $0.702 \pm 0.020$ & $116 / 12$ \\
\hline & $0.195 \pm 0.004$ & $2.40 \pm 0.02$ & $1.00 \pm 0.03$ & $1.987 \pm 0.060$ & $71 / 12$ \\
\hline & $0.208 \pm 0.003$ & $2.36 \pm 0.04$ & $0.70 \pm 0.03$ & $4.859 \pm 0.100$ & $88 / 9$ \\
\hline & $0.230 \pm 0.003$ & $2.10 \pm 0.02$ & $0.40 \pm 0.04$ & $8.318 \pm 0.120$ & $18 / 9$ \\
\hline \multirow{5}{*}{$\begin{array}{l}\text { Figure 2(b) } \\
0<z<18\end{array}$} & $0.260 \pm 0.004$ & $1.80 \pm 0.02$ & $0.40 \pm 0.02$ & $11.530 \pm 0.200$ & $33 / 9$ \\
\hline & $0.169 \pm 0.002$ & $2.14 \pm 0.05$ & $0.65 \pm 0.03$ & $13.579 \pm 0.200$ & $18 / 9$ \\
\hline & $0.168 \pm 0.004$ & $2.05 \pm 0.03$ & $0.30 \pm 0.03$ & $14.905 \pm 0.300$ & $27 / 6$ \\
\hline & $0.200 \pm 0.003$ & $1.60 \pm 0.02$ & $0.50 \pm 0.03$ & $14.783 \pm 0.300$ & $50 / 6$ \\
\hline & $0.175 \pm 0.003$ & $1.77 \pm 0.02$ & $0.35 \pm 0.04$ & $15.571 \pm 0.300$ & $37 / 6$ \\
\hline \multirow{5}{*}{$\begin{array}{l}\text { Figure } 2(\mathrm{c}) \\
0<z<18\end{array}$} & $0.210 \pm 0.030$ & $1.35 \pm 0.03$ & $0.25 \pm 0.02$ & $15.253 \pm 0.200$ & $39 / 6$ \\
\hline & $0.138 \pm 0.002$ & $2.00 \pm 0.04$ & $0.50 \pm 0.03$ & $27.457 \pm 0.400$ & $28 / 3$ \\
\hline & $0.138 \pm 0.002$ & $1.75 \pm 0.04$ & $0.60 \pm 0.03$ & $23.691 \pm 0.400$ & $38 / 2$ \\
\hline & $0.155 \pm 0.003$ & $1.05 \pm 0.02$ & $0.65 \pm 0.02$ & $21.444 \pm 0.400$ & $12 / 1$ \\
\hline & $0.160 \pm 0.003$ & $0.85 \pm 0.02$ & $0.75 \pm 0.03$ & $30.222 \pm 0.400$ & $2 /-$ \\
\hline \multirow{5}{*}{$\begin{array}{l}\text { Figure } 2 \text { (d) } \\
18<z<36\end{array}$} & $0.280 \pm 0.004$ & $2.40 \pm 0.03$ & $0.06 \pm 0.03$ & $0.313 \pm 0.020$ & $57 / 3$ \\
\hline & $0.138 \pm 0.002$ & $3.00 \pm 0.02$ & $1.52 \pm 0.03$ & $3.618 \pm 0.140$ & $73 / 12$ \\
\hline & $0.225 \pm 0.003$ & $2.30 \pm 0.02$ & $1.00 \pm 0.02$ & $11.850 \pm 0.200$ & $84 / 12$ \\
\hline & $0.208 \pm 0.003$ & $2.36 \pm 0.03$ & $0.70 \pm 0.03$ & $19.958 \pm 0.300$ & $69 / 9$ \\
\hline & $0.228 \pm 0.002$ & $2.10 \pm 0.01$ & $0.50 \pm 0.02$ & $24.746 \pm 0.400$ & $9 / 9$ \\
\hline \multirow{5}{*}{$\begin{array}{l}\text { Figure } 2(\mathrm{e}) \\
18<z<36\end{array}$} & $0.262 \pm 0.002$ & $1.70 \pm 0.02$ & $0.20 \pm 0.02$ & $25.438 \pm 0.300$ & $57 / 9$ \\
\hline & $0.182 \pm 0.004$ & $2.00 \pm 0.01$ & $0.65 \pm 0.03$ & $25.160 \pm 0.200$ & $64 / 9$ \\
\hline & $0.169 \pm 0.003$ & $2.05 \pm 0.03$ & $0.30 \pm 0.05$ & $24.000 \pm 0.300$ & $21 / 6$ \\
\hline & $0.200 \pm 0.005$ & $1.60 \pm 0.03$ & $0.40 \pm 0.02$ & $22.373 \pm 0.300$ & $30 / 6$ \\
\hline & $0.175 \pm 0.002$ & $1.77 \pm 0.01$ & $0.35 \pm 0.05$ & $21.727 \pm 0.400$ & $55 / 6$ \\
\hline \multirow{5}{*}{$\begin{array}{l}\text { Figure } 2 \text { (f) } \\
18<z<36\end{array}$} & $0.270 \pm 0.004$ & $1.00 \pm 0.02$ & $0.35 \pm 0.03$ & $19.006 \pm 0.200$ & $84 / 6$ \\
\hline & $0.139 \pm 0.003$ & $2.00 \pm 0.04$ & $0.50 \pm 0.03$ & $35.492 \pm 0.600$ & $28 / 3$ \\
\hline & $0.138 \pm 0.002$ & $1.75 \pm 0.05$ & $0.60 \pm 0.03$ & $31.840 \pm 0.600$ & $52 / 2$ \\
\hline & $0.155 \pm 0.003$ & $1.00 \pm 0.02$ & $0.65 \pm 0.02$ & $28.264 \pm 0.600$ & $7 / 1$ \\
\hline & $0.080 \pm 0.005$ & $1.40 \pm 0.02$ & $1.16 \pm 0.03$ & $23.464 \pm 0.600$ & $176 / 0$ \\
\hline \multirow{5}{*}{$\begin{array}{l}\text { Figure } 2 \text { (g) } \\
36<z<54\end{array}$} & $0.280 \pm 0.004$ & $2.40 \pm 0.03$ & $0.06 \pm 0.03$ & $0.690 \pm 0.020$ & $36 / 3$ \\
\hline & $0.149 \pm 0.003$ & $3.00 \pm 0.03$ & $1.52 \pm 0.04$ & $7.345 \pm 0.159$ & $95 / 12$ \\
\hline & $0.225 \pm 0.003$ & $2.45 \pm 0.03$ & $0.60 \pm 0.02$ & $16.629 \pm 0.360$ & $79 / 12$ \\
\hline & $0.208 \pm 0.002$ & $2.36 \pm 0.03$ & $0.60 \pm 0.03$ & $19.895 \pm 0.300$ & $58 / 9$ \\
\hline & $0.218 \pm 0.002$ & $2.10 \pm 0.02$ & $0.30 \pm 0.03$ & $20.966 \pm 0.400$ & $33 / 9$ \\
\hline \multirow{5}{*}{$\begin{array}{l}\text { Figure } 2 \text { (h) } \\
36<z<54\end{array}$} & $0.260 \pm 0.003$ & $1.70 \pm 0.02$ & $0.20 \pm 0.02$ & $22.186 \pm 0.400$ & $48 / 9$ \\
\hline & $0.182 \pm 0.002$ & $2.00 \pm 0.03$ & $0.55 \pm 0.03$ & $20.936 \pm 0.360$ & $63 / 9$ \\
\hline & $0.170 \pm 0.002$ & $2.05 \pm 0.03$ & $0.30 \pm 0.03$ & $20.232 \pm 0.300$ & $25 / 6$ \\
\hline & $0.210 \pm 0.004$ & $1.60 \pm 0.04$ & $0.40 \pm 0.03$ & $17.743 \pm 0.200$ & $9 / 6$ \\
\hline & $0.175 \pm 0.002$ & $1.77 \pm 0.01$ & $0.35 \pm 0.02$ & $18.830 \pm 0.300$ & $41 / 6$ \\
\hline
\end{tabular}


TABLe 2: Continued.

\begin{tabular}{|c|c|c|c|c|c|}
\hline Figure & $T(\mathrm{GeV})$ & $y_{\max }$ & $y_{\min }$ & $N_{0}(\times 0.001)$ & $\chi^{2} /$ ndof \\
\hline \multirow{5}{*}{$\begin{array}{l}\text { Figure 2(i) } \\
36<z<54\end{array}$} & $0.270 \pm 0.002$ & $1.00 \pm 0.01$ & $0.15 \pm 0.01$ & $16.232 \pm 0.200$ & $74 / 6$ \\
\hline & $0.140 \pm 0.002$ & $2.00 \pm 0.01$ & $0.50 \pm 0.02$ & $31.730 \pm 0.640$ & $30 / 3$ \\
\hline & $0.138 \pm 0.002$ & $1.75 \pm 0.02$ & $0.40 \pm 0.02$ & $24.610 \pm 0.400$ & $47 / 2$ \\
\hline & $0.155 \pm 0.002$ & $1.05 \pm 0.02$ & $0.70 \pm 0.03$ & $23.472 \pm 0.600$ & $9 / 1$ \\
\hline & $0.090 \pm 0.003$ & $1.40 \pm 0.02$ & $0.16 \pm 0.01$ & $19.198 \pm 0.400$ & $8 / 0$ \\
\hline \multirow{5}{*}{$\begin{array}{l}\text { Figure } 2(j) \\
54<z<72\end{array}$} & $0.280 \pm 0.004$ & $2.50 \pm 0.03$ & $0.06 \pm 0.03$ & $0.943 \pm 0.020$ & $41 / 3$ \\
\hline & $0.149 \pm 0.003$ & $3.05 \pm 0.02$ & $1.52 \pm 0.03$ & $7.362 \pm 0.200$ & $65 / 12$ \\
\hline & $0.225 \pm 0.004$ & $2.40 \pm 0.02$ & $0.60 \pm 0.02$ & $13.744 \pm 0.200$ & $61 / 12$ \\
\hline & $0.208 \pm 0.003$ & $2.36 \pm 0.01$ & $0.60 \pm 0.03$ & $15.998 \pm 0.200$ & $60 / 9$ \\
\hline & $0.220 \pm 0.003$ & $2.08 \pm 0.02$ & $0.30 \pm 0.03$ & $16.243 \pm 0.240$ & $13 / 9$ \\
\hline \multirow{5}{*}{$\begin{array}{l}\text { Figure } 2(\mathrm{k}) \\
54<z<72\end{array}$} & $0.260 \pm 0.004$ & $1.70 \pm 0.02$ & $0.30 \pm 0.02$ & $16.868 \pm 0.240$ & $50 / 9$ \\
\hline & $0.184 \pm 0.002$ & $2.00 \pm 0.02$ & $0.55 \pm 0.03$ & $15.986 \pm 0.200$ & $70 / 9$ \\
\hline & $0.170 \pm 0.003$ & $2.05 \pm 0.03$ & $0.20 \pm 0.02$ & $15.533 \pm 0.200$ & $28 / 6$ \\
\hline & $0.210 \pm 0.004$ & $1.55 \pm 0.02$ & $0.20 \pm 0.03$ & $13.369 \pm 0.200$ & $48 / 6$ \\
\hline & $0.175 \pm 0.003$ & $1.77 \pm 0.03$ & $0.35 \pm 0.02$ & $14.123 \pm 0.200$ & $25 / 6$ \\
\hline \multirow{5}{*}{$\begin{array}{l}\text { Figure 2(l) } \\
54<z<72\end{array}$} & $0.280 \pm 0.004$ & $1.00 \pm 0.03$ & $0.15 \pm 0.03$ & $12.808 \pm 0.100$ & $66 / 6$ \\
\hline & $0.140 \pm 0.002$ & $2.00 \pm 0.02$ & $0.40 \pm 0.02$ & $23.940 \pm 0.400$ & $31 / 3$ \\
\hline & $0.138 \pm 0.002$ & $1.75 \pm 0.03$ & $0.40 \pm 0.04$ & $19.141 \pm 0.400$ & $44 / 2$ \\
\hline & $0.155 \pm 0.002$ & $1.05 \pm 0.03$ & $0.70 \pm 0.02$ & $15.698 \pm 0.400$ & $16 / 1$ \\
\hline & $0.135 \pm 0.003$ & $2.70 \pm 0.02$ & $0.66 \pm 0.02$ & $15.298 \pm 0.400$ & $26 / 1$ \\
\hline \multirow{5}{*}{$\begin{array}{l}\text { Figure } 2(\mathrm{~m}) \\
72<z<90\end{array}$} & $0.280 \pm 0.002$ & $2.50 \pm 0.03$ & $0.06 \pm 0.02$ & $1.019 \pm 0.020$ & $43 / 3$ \\
\hline & $0.149 \pm 0.003$ & $3.05 \pm 0.03$ & $1.52 \pm 0.03$ & $6.509 \pm 0.240$ & $73 / 12$ \\
\hline & $0.225 \pm 0.002$ & $2.37 \pm 0.02$ & $0.60 \pm 0.03$ & $9.724 \pm 0.200$ & $88 / 12$ \\
\hline & $0.208 \pm 0.002$ & $2.30 \pm 0.03$ & $0.40 \pm 0.03$ & $11.638 \pm 0.200$ & $86 / 9$ \\
\hline & $0.220 \pm 0.002$ & $2.02 \pm 0.02$ & $0.25 \pm 0.02$ & $12.192 \pm 0.200$ & $23 / 9$ \\
\hline \multirow{5}{*}{$\begin{array}{l}\text { Figure } 2(\mathrm{n}) \\
72<z<90\end{array}$} & $0.260 \pm 0.003$ & $1.60 \pm 0.02$ & $0.30 \pm 0.02$ & $12.207 \pm 0.200$ & $51 / 9$ \\
\hline & $0.188 \pm 0.002$ & $1.90 \pm 0.03$ & $0.55 \pm 0.03$ & $11.864 \pm 0.200$ & $33 / 9$ \\
\hline & $0.170 \pm 0.003$ & $2.05 \pm 0.02$ & $0.20 \pm 0.03$ & $11.571 \pm 0.200$ & $7 / 6$ \\
\hline & $0.210 \pm 0.003$ & $1.55 \pm 0.02$ & $0.20 \pm 0.02$ & $11.074 \pm 0.200$ & $45 / 6$ \\
\hline & $0.175 \pm 0.003$ & $1.77 \pm 0.02$ & $0.35 \pm 0.03$ & $11.769 \pm 0.200$ & $41 / 6$ \\
\hline \multirow{5}{*}{$\begin{array}{l}\text { Figure } 2(\text { o) } \\
72<z<90\end{array}$} & $0.280 \pm 0.003$ & $1.00 \pm 0.03$ & $0.15 \pm 0.04$ & $10.322 \pm 0.200$ & $50 / 6$ \\
\hline & $0.140 \pm 0.003$ & $2.00 \pm 0.04$ & $0.30 \pm 0.03$ & $18.405 \pm 0.400$ & $23 / 3$ \\
\hline & $0.138 \pm 0.004$ & $1.75 \pm 0.04$ & $0.40 \pm 0.05$ & $15.381 \pm 0.400$ & $30 / 2$ \\
\hline & $0.155 \pm 0.004$ & $1.05 \pm 0.03$ & $0.70 \pm 0.03$ & $12.841 \pm 0.600$ & $15 / 1$ \\
\hline & $0.135 \pm 0.002$ & $2.70 \pm 0.04$ & $0.06 \pm 0.04$ & $11.641 \pm 0.400$ & $19 / 1$ \\
\hline \multirow{5}{*}{$\begin{array}{l}\text { Figure } 2(\mathrm{p}) \\
z=90\end{array}$} & $0.280 \pm 0.006$ & $2.60 \pm 0.04$ & $0.06 \pm 0.01$ & $9.687 \pm 0.200$ & $47 / 3$ \\
\hline & $0.147 \pm 0.002$ & $3.10 \pm 0.02$ & $1.32 \pm 0.02$ & $14.156 \pm 0.140$ & $18 / 12$ \\
\hline & $0.225 \pm 0.003$ & $2.32 \pm 0.03$ & $0.60 \pm 0.03$ & $11.283 \pm 0.200$ & $70 / 12$ \\
\hline & $0.208 \pm 0.002$ & $2.30 \pm 0.02$ & $0.40 \pm 0.03$ & $10.248 \pm 0.160$ & $66 / 9$ \\
\hline & $0.220 \pm 0.003$ & $2.00 \pm 0.02$ & $0.30 \pm 0.02$ & $8.134 \pm 0.200$ & $64 / 9$ \\
\hline
\end{tabular}


TABle 2: Continued.

\begin{tabular}{lccccc}
\hline Figure & $T(\mathrm{GeV})$ & $y_{\max }$ & $y_{\min }$ & $N_{0}(\times 0.001)$ & $12.255 \pm 0.400$ \\
\hline & $0.260 \pm 0.004$ & $1.55 \pm 0.01$ & $0.30 \pm 0.02$ & $8.593 \pm 0.400$ & $31 / 9$ \\
Figure 2(q) & $0.188 \pm 0.004$ & $1.80 \pm 0.02$ & $0.25 \pm 0.02$ & $5.816 \pm 0.320$ \\
$z=90$ & $0.165 \pm 0.002$ & $1.80 \pm 0.03$ & $0.20 \pm 0.03$ & $4.394 \pm 0.120$ & $37 / 6$ \\
& $0.210 \pm 0.005$ & $1.30 \pm 0.01$ & $0.10 \pm 0.03$ & $3.019 \pm 0.040$ \\
\hline
\end{tabular}
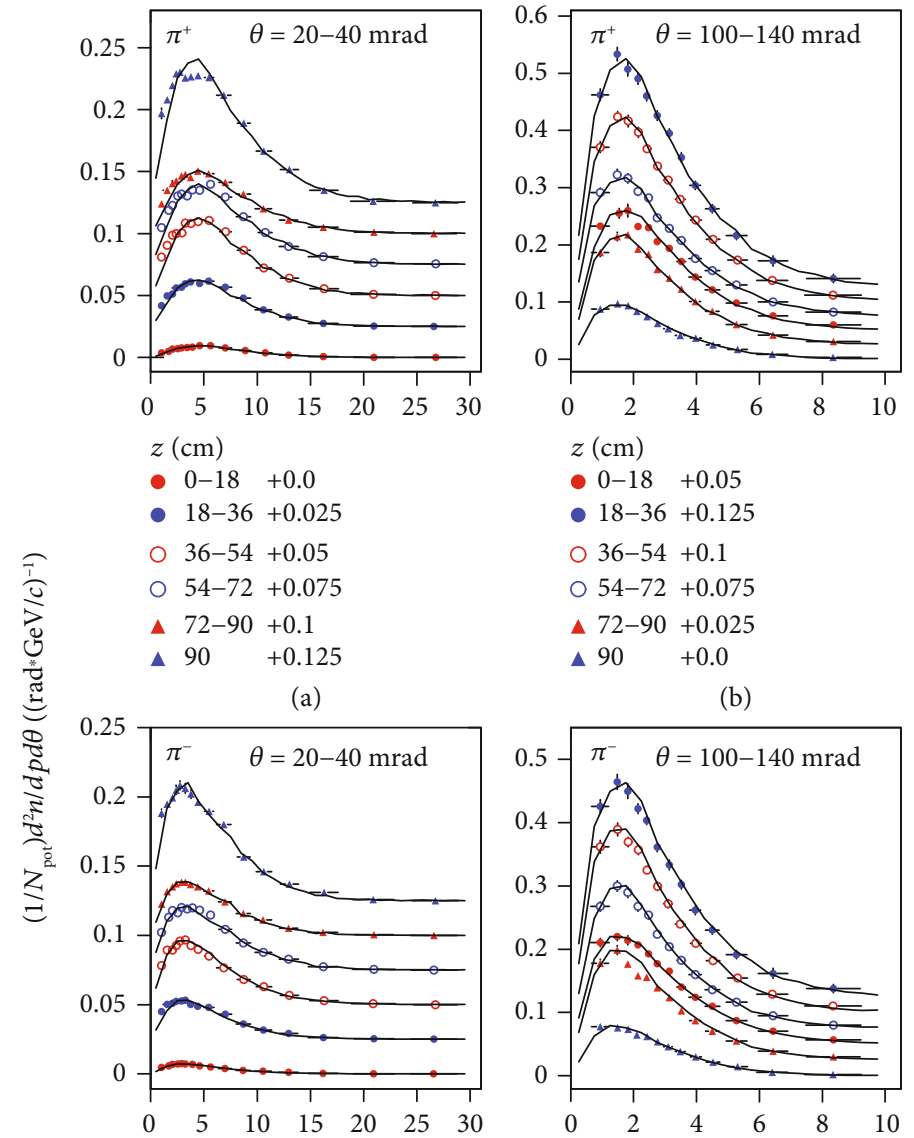

o $36-54+0.1$

○ $54-72+0.075$

72-90+0.025

$\Delta 90+0.0$

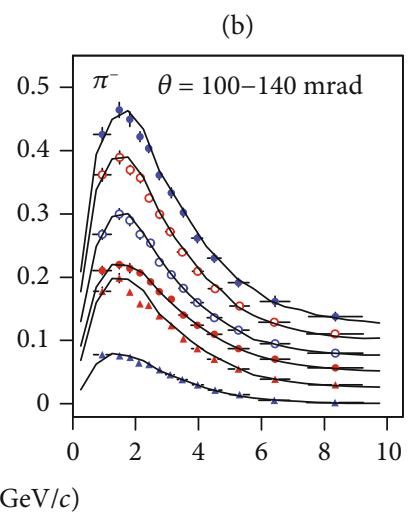

$$
\begin{array}{lll}
z(\mathrm{~cm}) & \\
\bullet & 0-18 & +0.0 \\
- & 18-36 & +0.025 \\
0 & 36-54 & +0.05 \\
0 & 54-72 & +0.075 \\
\text { × } & 72-90 & +0.1 \\
\triangle & 90 \quad+0.125
\end{array}
$$

$z(\mathrm{~cm})$

- $0-18+0.05$

- $18-36+0.125$

○ $36-54+0.1$

○ $54-72+0.075$

$\triangle 72-90+0.025$

$\triangle 90+0.0$

(c)

(d)

Figure 3: Same as Figure 1 but showing the spectra of $(\mathrm{a}-\mathrm{b}) \pi^{+}$and $(\mathrm{c}-\mathrm{d}) \pi^{-}$in $(\mathrm{a}-\mathrm{c}) \theta=20-40 \mathrm{mrad}$ and $(\mathrm{b}-\mathrm{d}) \theta=100-140 \mathrm{mrad}$ in six $z$ ranges. 


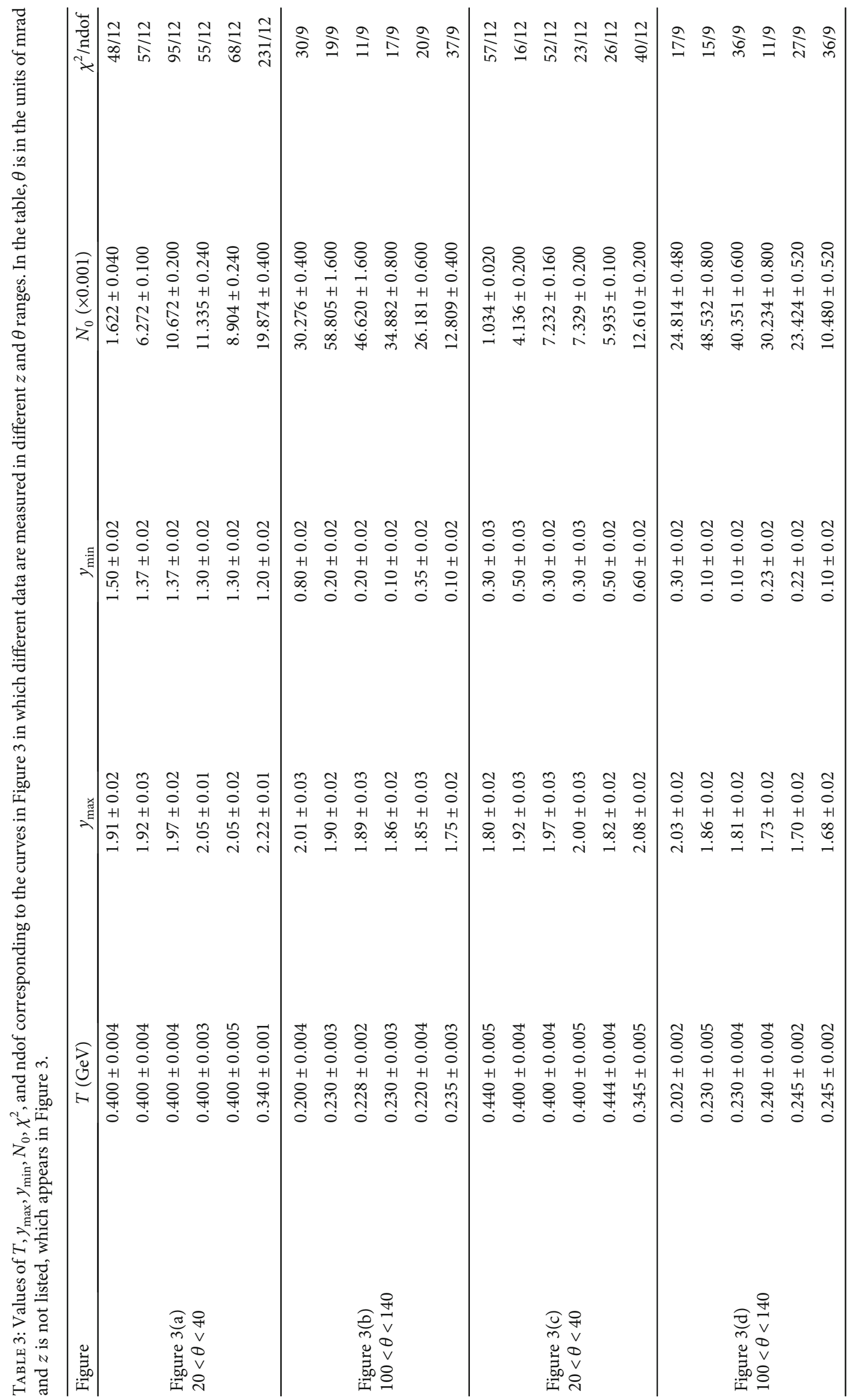




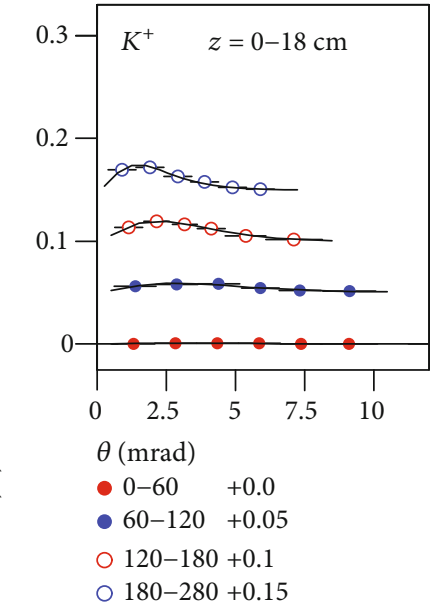

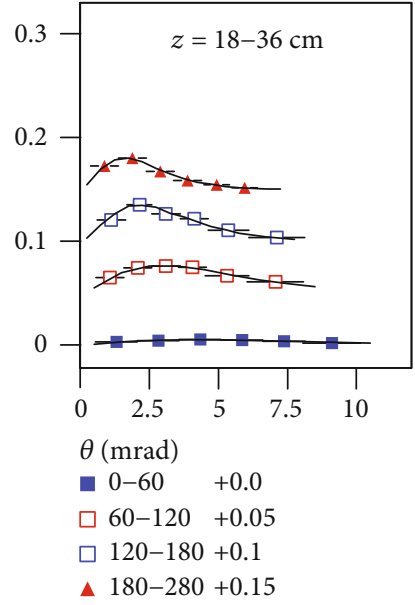

(b)

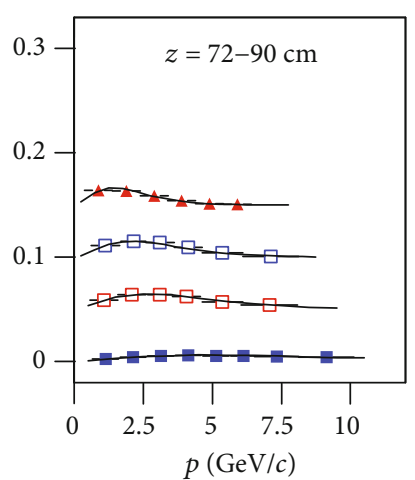

$\theta(\mathrm{mrad})$

a $0-60+0.0$

$\square 60-120+0.05$

$\square 120-180+0.1$

A $180-280+0.15$

(e)

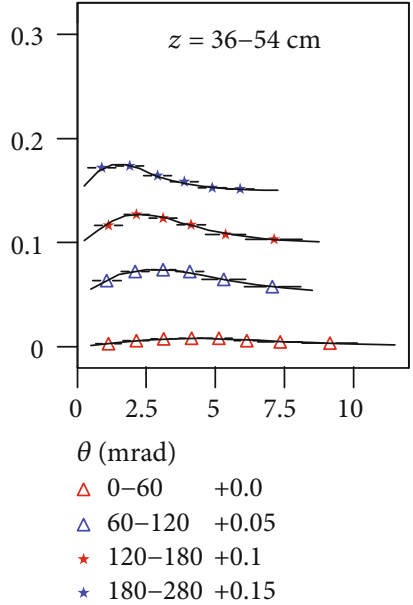

(c)

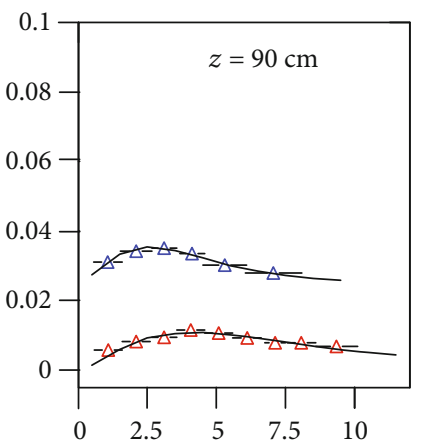

$\theta(\mathrm{mrad})$

$\triangle 0-60+0.0$

$\triangle 60-120+0.025$

$60-120+0.05$

○ $180-280+0.15$

(d)

(f)

Figure 4: Same as Figure 1 but showing the spectra of $K^{+}$. Panels (a-f) represent the spectra for $z=0-18,18-36,36-54,54-72,72-90$, and $90 \mathrm{~cm}$, respectively.

Under the assumption of isotropic emission in the rest frame of emission source, the emission angle $\theta^{\prime}$ of the considered particle has the probability density function:

$$
f_{\theta^{\prime}}\left(\theta^{\prime}\right)=\frac{1}{2} \sin \theta^{\prime}
$$

which is a half sine distribution in $[0, \pi]$, and the azimuth $\varphi^{\prime}$ obeys the probability density function $f_{\varphi^{\prime}}\left(\varphi^{\prime}\right)=1 /(2 \pi)$ which is an even distribution in $[0,2 \pi]$ [41]. In the Monte Carlo method, $\theta^{\prime}$ satisfies

$$
\theta^{\prime}=2 \arcsin \left(\sqrt{R_{2}}\right)
$$

which is the solution of $\int_{0}^{\theta^{\prime}}(1 / 2) \sin \theta^{\prime \prime} d \theta^{\prime \prime}=R_{2}$.
Considering $p^{\prime}$ and $\theta^{\prime}$ obtained from Equations (3) and (5), we have the transverse momentum $p_{T}^{\prime}$ to be

$$
p_{T}^{\prime}=p^{\prime} \sin \theta^{\prime}
$$

the longitudinal momentum $p_{z}^{\prime}$ to be

$$
p_{z}^{\prime}=p^{\prime} \cos \theta^{\prime}
$$

the energy $E^{\prime}$ to be

$$
E^{\prime}=\sqrt{p^{\prime 2}+m_{0}^{2}},
$$

and the rapidity $y^{\prime}$ to be

$$
y^{\prime} \equiv \frac{1}{2} \ln \left(\frac{E^{\prime}+p_{z}^{\prime}}{E^{\prime}-p_{z}^{\prime}}\right) .
$$




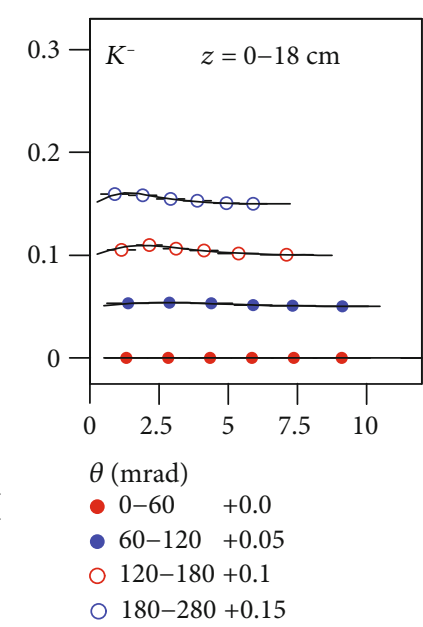

(a)

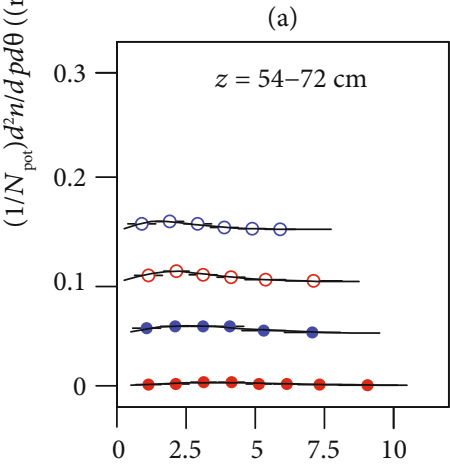

$\begin{array}{ll}\theta(\mathrm{mrad}) & \\ -0-60+0.0 \\ \text { - } & 60-120+0.05 \\ 0 & 120-180+0.1 \\ \text { } & 180-280+0.15\end{array}$

(d)

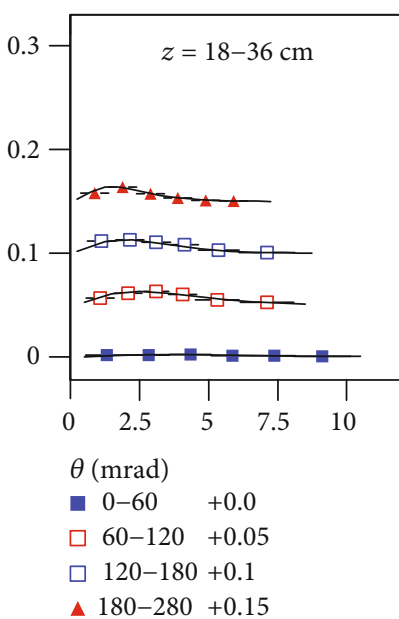

(b)

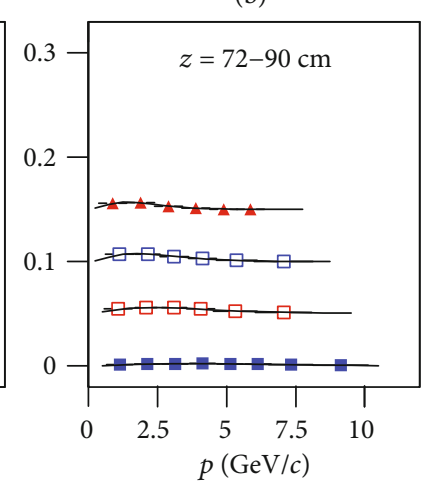

$\theta(\mathrm{mrad})$

a $0-60+0.0$

$\square 60-120+0.05$

$\square 120-180+0.1$

^ 180-280+0.15

(e)

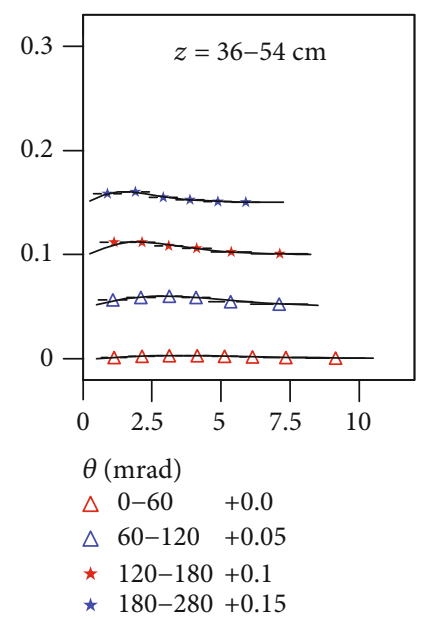

(c)

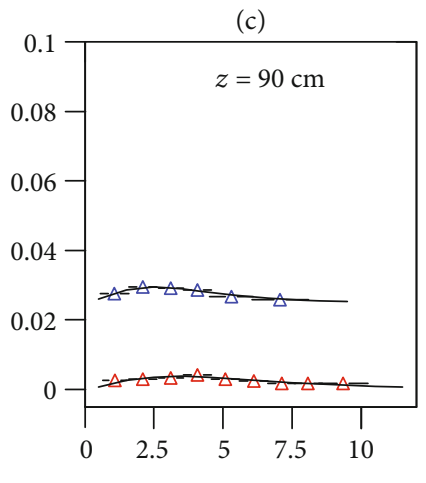

$\theta(\mathrm{mrad})$

$\triangle 0-60+0.0$

$\triangle 60-120+0.025$

Figure 5: Same as Figure 1 but showing the spectra of $K^{-}$. Panels (a-f) represent the spectra for $z=0-18,18-36,36-54,54-72,72-90$, and $90 \mathrm{~cm}$, respectively.

In the center-of-mass reference frame or the laboratory reference frame, the rapidity of the considered emission source is assumed to be $y_{x}$ in the rapidity space. Then, the rapidity of the considered particle in the center-of-mass or laboratory reference frame is

$$
y=y^{\prime}+y_{x}
$$

due to the additivity of rapidity. Multiple emission sources are assumed to distribute evenly in the rapidity range $\left[y_{\text {min }}\right.$, $\left.y_{\max }\right]$, where $y_{\min }$ and $y_{\max }$ are the minimum and maximum rapidity shifts of the multiple sources, respectively. In the Monte Carlo method,

$$
y_{x}=\left(y_{\max }-y_{\min }\right) R_{3}+y_{\min } .
$$

In particular, comparing with small mass particles, protons exhibit large effect of leading particles which are assumed to distribute evenly in the rapidity range
$\left[y_{L \min }, y_{L \max }\right]$, where $y_{L \text { min }}$ and $y_{L \text { max }}$ are the minimum and maximum rapidity shifts of the leading protons, respectively. We have

$$
y_{x}=\left(y_{L \max }-y_{L \min }\right) R_{4}+y_{L \min } .
$$

The fraction of the nonleading (leading) protons in total protons is assumed to be $k(1-k)$. The effects of leading pions and kaons are small and can be neglected in this paper.

In the center-of-mass or laboratory reference frame, the transverse momentum $p_{T}$ is

$$
p_{T}=p_{T}^{\prime}
$$

the longitudinal momentum $p_{z}$ is

$$
p_{z}=\sqrt{p_{T}^{2}+m_{0}^{2}} \sinh y
$$


TABLe 4: Values of $T, y_{\max }, y_{\min }, N_{0}, \chi^{2}$, and ndof corresponding to the curves in Figure 4 in which different data are measured in different $\theta$ and $z$ ranges. In the table, $z$ is in the units of $\mathrm{cm}$ and $\theta$ is not listed, which appears in Figure 4.

\begin{tabular}{|c|c|c|c|c|c|}
\hline Figure & $T(\mathrm{GeV})$ & $y_{\max }$ & $y_{\min }$ & $N_{0}(\times 0.001)$ & $\chi^{2} /$ ndof \\
\hline \multirow{4}{*}{$\begin{array}{l}\text { Figure 4(a) } \\
0<z<18\end{array}$} & $0.300 \pm 0.003$ & $2.00 \pm 0.03$ & $1.20 \pm 0.03$ & $0.335 \pm 0.012$ & $18 / 2$ \\
\hline & $0.300 \pm 0.003$ & $1.90 \pm 0.02$ & $1.10 \pm 0.03$ & $3.079 \pm 0.120$ & $30 / 2$ \\
\hline & $0.300 \pm 0.003$ & $1.45 \pm 0.03$ & $1.10 \pm 0.03$ & $4.787 \pm 0.180$ & $24 / 2$ \\
\hline & $0.300 \pm 0.004$ & $1.10 \pm 0.02$ & $0.60 \pm 0.03$ & $6.462 \pm 0.300$ & $31 / 2$ \\
\hline \multirow{4}{*}{$\begin{array}{l}\text { Figure } 4 \text { (b) } \\
18<z<36\end{array}$} & $0.400 \pm 0.003$ & $2.00 \pm 0.03$ & $1.20 \pm 0.03$ & $2.569 \pm 0.060$ & $8 / 2$ \\
\hline & $0.300 \pm 0.003$ & $2.00 \pm 0.03$ & $1.10 \pm 0.02$ & $9.552 \pm 0.300$ & $26 / 2$ \\
\hline & $0.400 \pm 0.004$ & $1.00 \pm 0.02$ & $0.95 \pm 0.01$ & $8.080 \pm 0.180$ & $27 / 2$ \\
\hline & $0.320 \pm 0.004$ & $1.08 \pm 0.02$ & $0.70 \pm 0.03$ & $8.773 \pm 0.100$ & $25 / 2$ \\
\hline \multirow{4}{*}{$\begin{array}{l}\text { Figure } 4(\mathrm{c}) \\
36<z<54\end{array}$} & $0.420 \pm 0.004$ & $1.75 \pm 0.02$ & $1.35 \pm 0.02$ & $3.828 \pm 0.060$ & $47 / 4$ \\
\hline & $0.300 \pm 0.004$ & $1.85 \pm 0.04$ & $1.10 \pm 0.03$ & $7.922 \pm 0.180$ & $39 / 2$ \\
\hline & $0.280 \pm 0.004$ & $1.45 \pm 0.04$ & $1.35 \pm 0.03$ & $6.561 \pm 0.240$ & $21 / 2$ \\
\hline & $0.316 \pm 0.004$ & $1.15 \pm 0.03$ & $0.40 \pm 0.04$ & $7.016 \pm 0.500$ & $14 / 2$ \\
\hline \multirow{4}{*}{$\begin{array}{l}\text { Figure } 4(\mathrm{~d}) \\
54<z<72\end{array}$} & $0.400 \pm 0.005$ & $2.00 \pm 0.03$ & $1.30 \pm 0.02$ & $4.065 \pm 0.180$ & $38 / 4$ \\
\hline & $0.300 \pm 0.003$ & $1.80 \pm 0.03$ & $1.05 \pm 0.03$ & $5.934 \pm 0.300$ & $18 / 2$ \\
\hline & $0.360 \pm 0.005$ & $1.35 \pm 0.03$ & $0.60 \pm 0.06$ & $4.578 \pm 0.180$ & $13 / 2$ \\
\hline & $0.275 \pm 0.002$ & $1.02 \pm 0.01$ & $1.00 \pm 0.01$ & $5.361 \pm 0.200$ & $38 / 2$ \\
\hline \multirow{4}{*}{$\begin{array}{l}\text { Figure } 4(\mathrm{e}) \\
72<z<90\end{array}$} & $0.400 \pm 0.004$ & $2.20 \pm 0.03$ & $1.35 \pm 0.03$ & $3.806 \pm 0.120$ & $14 / 4$ \\
\hline & $0.300 \pm 0.004$ & $1.80 \pm 0.03$ & $1.05 \pm 0.03$ & $4.641 \pm 0.120$ & $32 / 2$ \\
\hline & $0.280 \pm 0.003$ & $1.45 \pm 0.03$ & $1.20 \pm 0.03$ & $3.491 \pm 0.120$ & $17 / 2$ \\
\hline & $0.300 \pm 0.004$ & $1.10 \pm 0.03$ & $0.50 \pm 0.04$ & $4.315 \pm 0.150$ & $22 / 2$ \\
\hline \multirow{2}{*}{$\begin{array}{l}\text { Figure } 4(\mathrm{f}) \\
z=90\end{array}$} & $0.400 \pm 0.003$ & $2.20 \pm 0.03$ & $1.00 \pm 0.03$ & $6.300 \pm 0.120$ & $10 / 5$ \\
\hline & $0.300 \pm 0.006$ & $1.79 \pm 0.03$ & $1.00 \pm 0.02$ & $3.123 \pm 0.060$ & $36 / 2$ \\
\hline
\end{tabular}

the momentum $p$ is

$$
p=\sqrt{p_{T}^{2}+p_{z}^{2}}
$$

and the emission angle $\theta$ is

$$
\theta=\arctan \left(\frac{p_{T}}{p_{z}}\right) .
$$

The whole calculation is performed by the Monte Carlo method, though only random numbers are used for the numerical calculation. To compare the theoretical model results with the experimental momentum spectra in a given $\theta$ range, we analyze the momentum distribution of particles which are in the given $\theta$ range. It should be noted that another experimental selection, i.e., the longitudinal position $z$ [32], is not regarded as the selected condition in the theoretical model work due to the fact that $z$ is only a reflection of target thickness in a $90 \mathrm{~cm}$ long graphite target. From $z=0$ to $z=90 \mathrm{~cm}$, the beam momentum slightly decreases, which is neglected in this paper. In the calculation using random numbers, the energy-momentum conservation was demanded at each step. The results violating the energy-momentum conservation are not considered for our discussions.

It should be noticed that the Boltzmann distribution, Equation (1), can be used to describe low momentum spectra in the source's rest frame or low transverse momentum spectra after analytic derivation [41] or via the Monte Carlo method, Equations (3), (5), and (6). In the case of considering high momentum spectra in the source's rest frame or high transverse momentum spectra, one may use possibly the multicomponent Boltzmann distribution, Equation (2). This paper treats multiple sources moving directly in a rapidity range, $\left[y_{\min }, y_{\max }\right]$ or $\left[y_{L \text { min }}, y_{L \text { max }}\right]$, which results in high momentum in the laboratory reference frame. However, in the rest frame of each source, the total momentum and transverse momentum are small. As a consequence, Equation (1) is valid in all momentum ranges, after the transformation from the source's rest frame to the laboratory reference frame.

\section{Results and Discussion}

Figures 1 and 2 present the momentum spectra, $\left(1 / N_{\text {pot }}\right)$ $d^{2} n / d p d \theta$, of charged pions $\left(\pi^{+}\right.$and $\left.\pi^{-}\right)$produced in $p$ - 


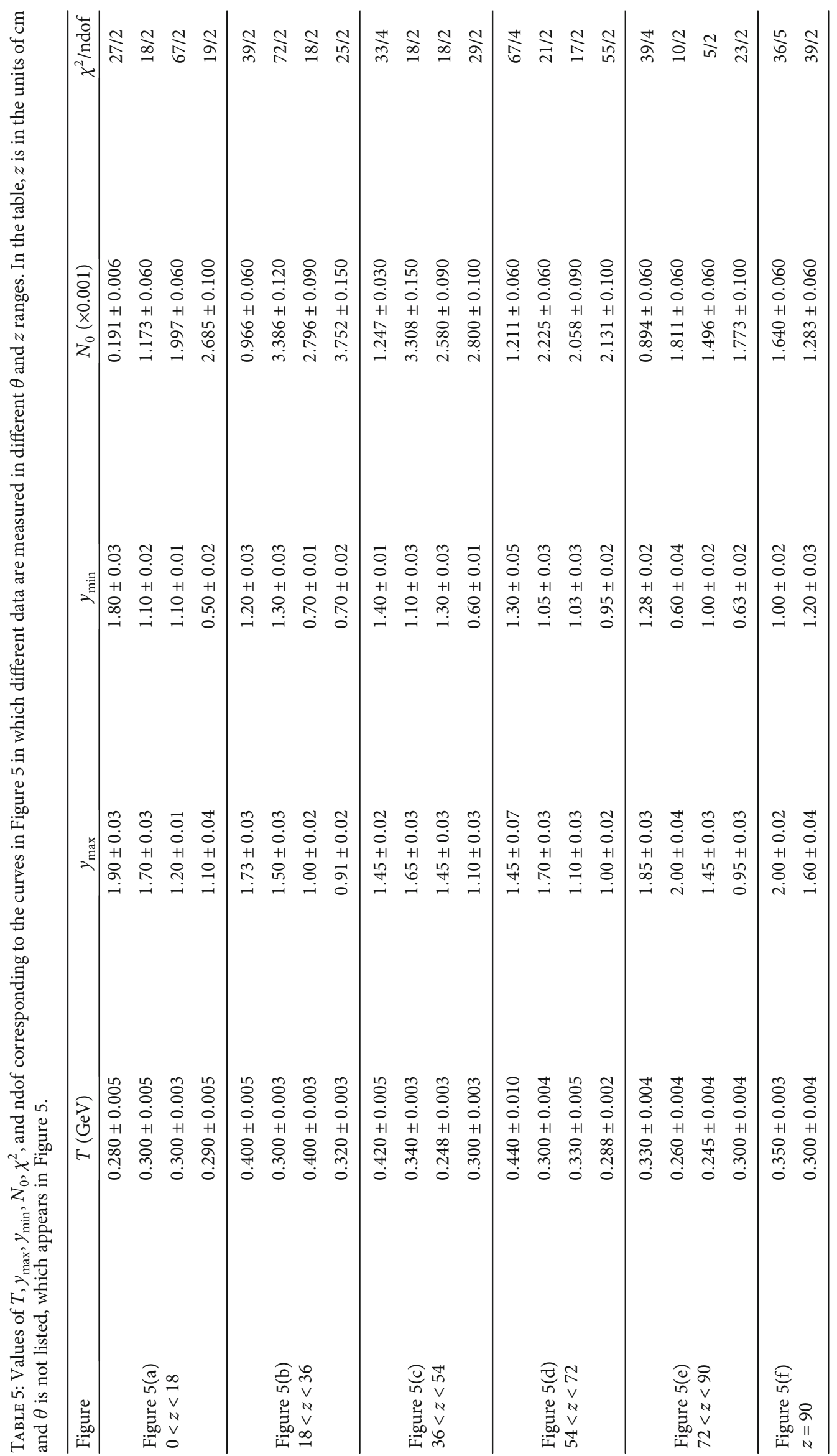



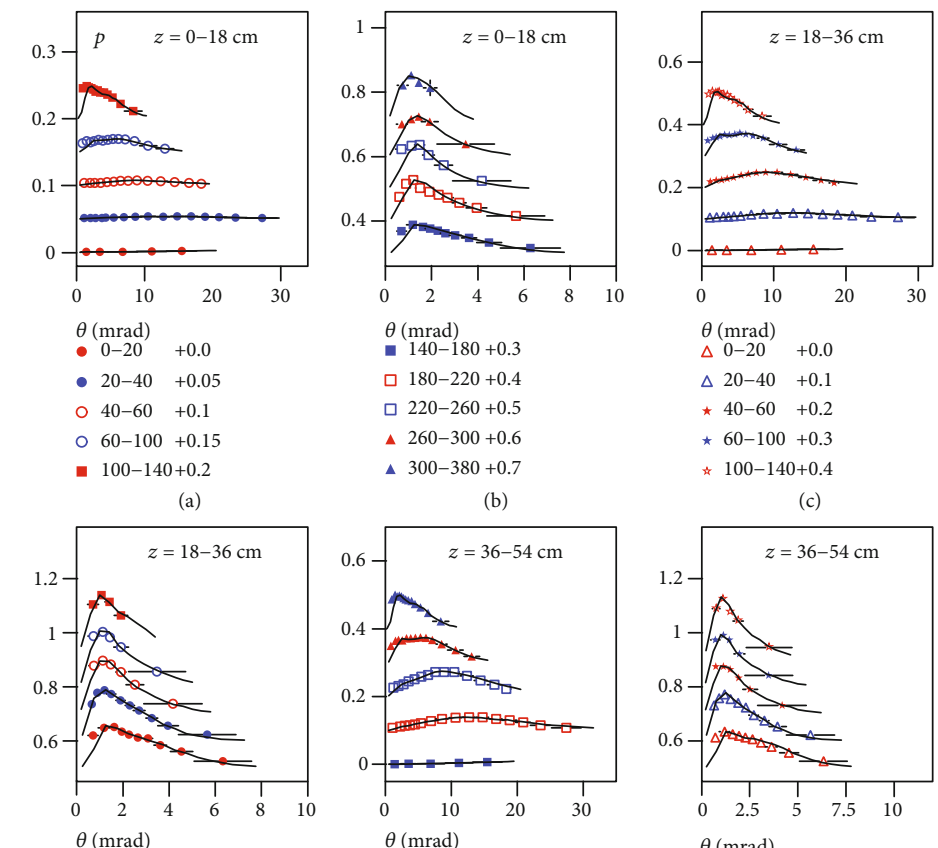

(c)
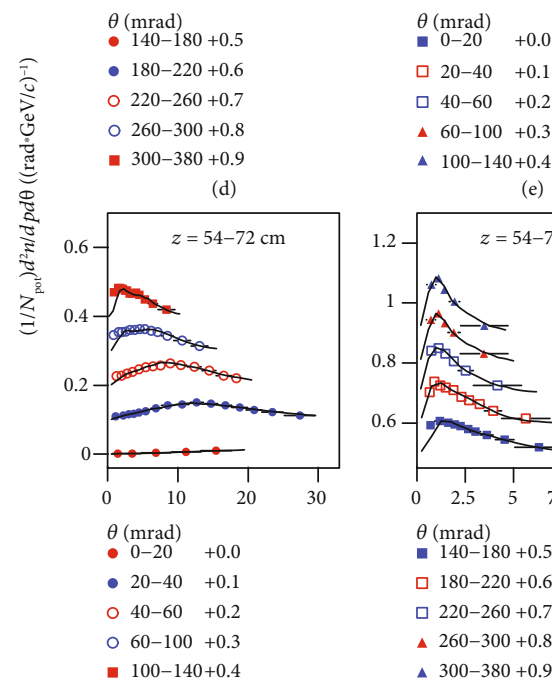

ㅁ $20-40 \quad+0.1$

ㅁ 40-60 +0.2

\ $60-100+0.3$

- $100-140+0.4$

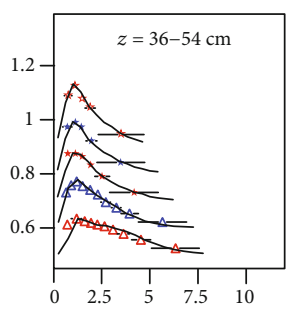

$\theta$ (mrad)

$\triangle 140-180+0.5$

$\triangle 180-220+0.6$

$\star 220-260+0.7$

^ 260-300+0.8

\& $300-380+0.9$
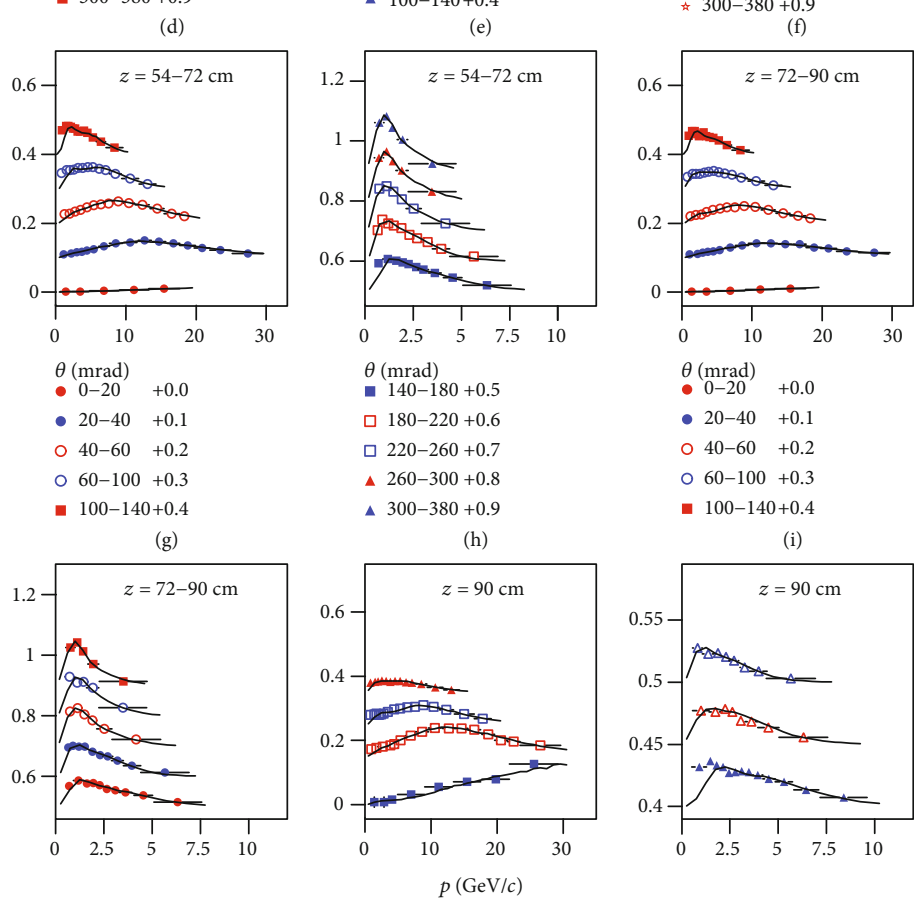

$\theta$ (mrad)

- $140-180+0.5$

$\theta$ (mrad)

- $0-20+0.0$

$\theta$ (mrad)

- $180-220+0.6$

ㅁ $20-40+0.15$

\ $100-140+0.4$

○ $220-260+0.7$

- $260-300+0.8$

- $300-380+0.9$

ㅁ $40-60+0.25$

$\triangle 140-180+0.45$

$\Delta 180-220+0.5$

(j)

(1)

Figure 6: Same as Figure 1 but showing the spectra of $p$. Panels (a, b), (c, d), (e, f), (g, h), (i, j), and (k, l) represent the spectra for $z=0-18,18-$ $36,36-54,54-72,72-90$, and $90 \mathrm{~cm}$, respectively. 


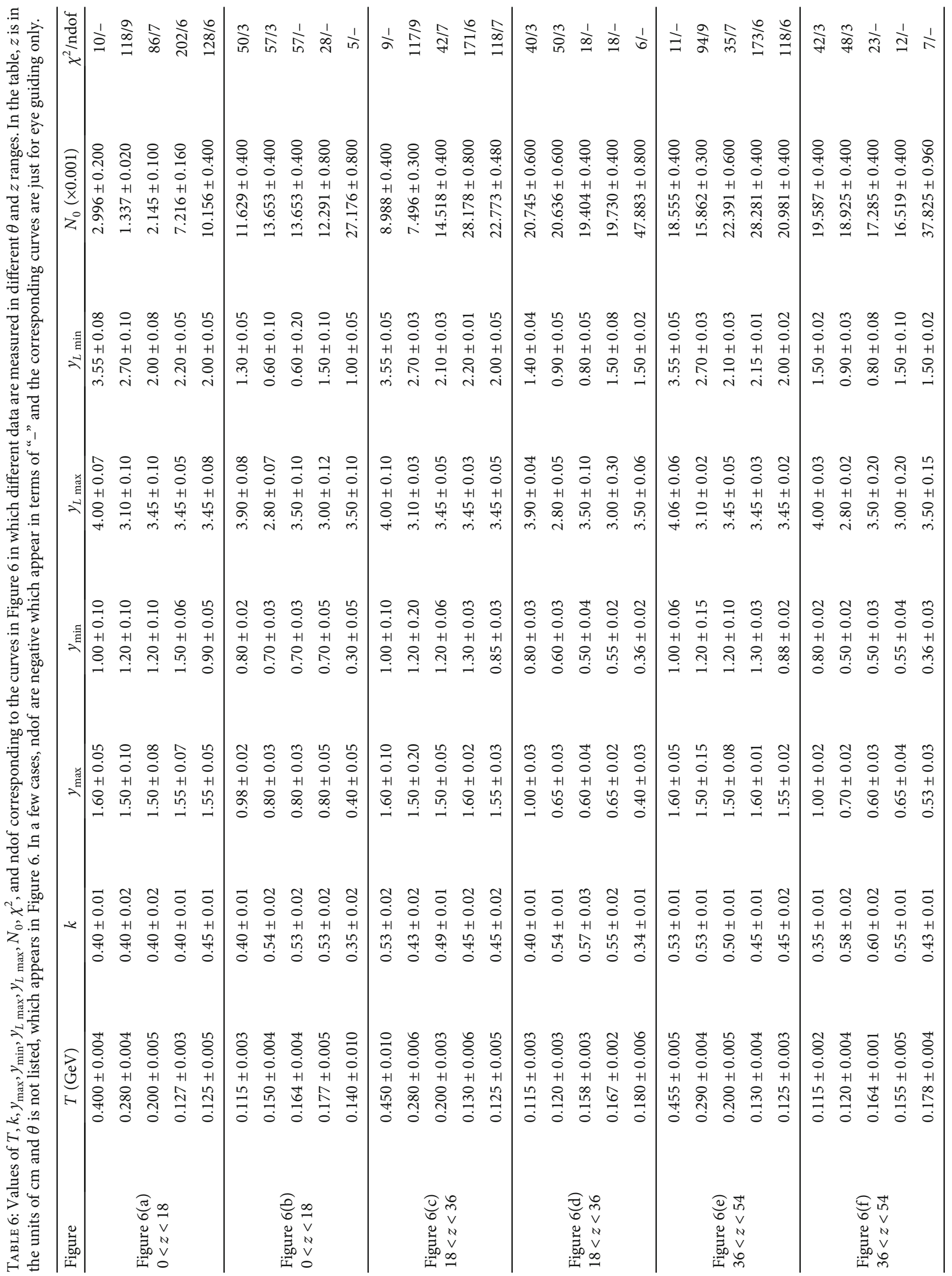




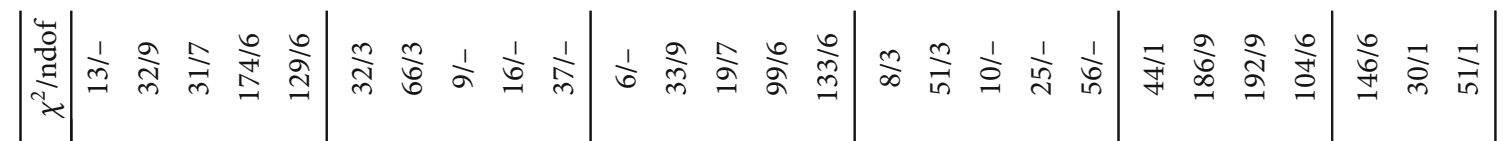

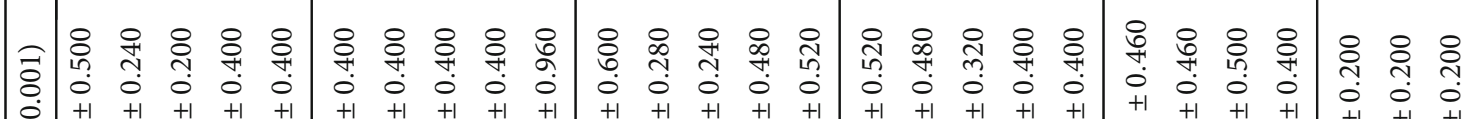

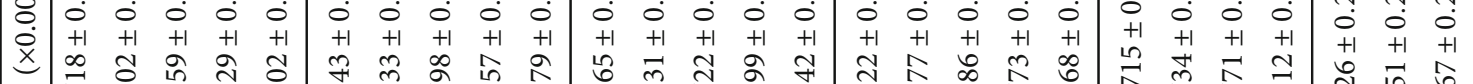

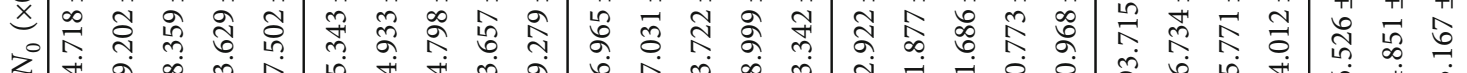

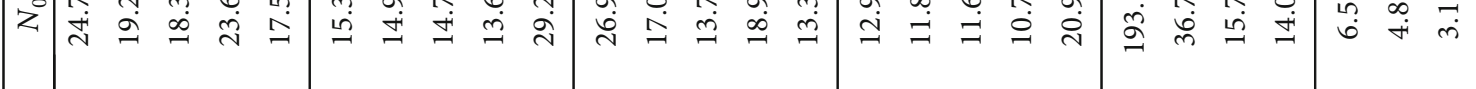

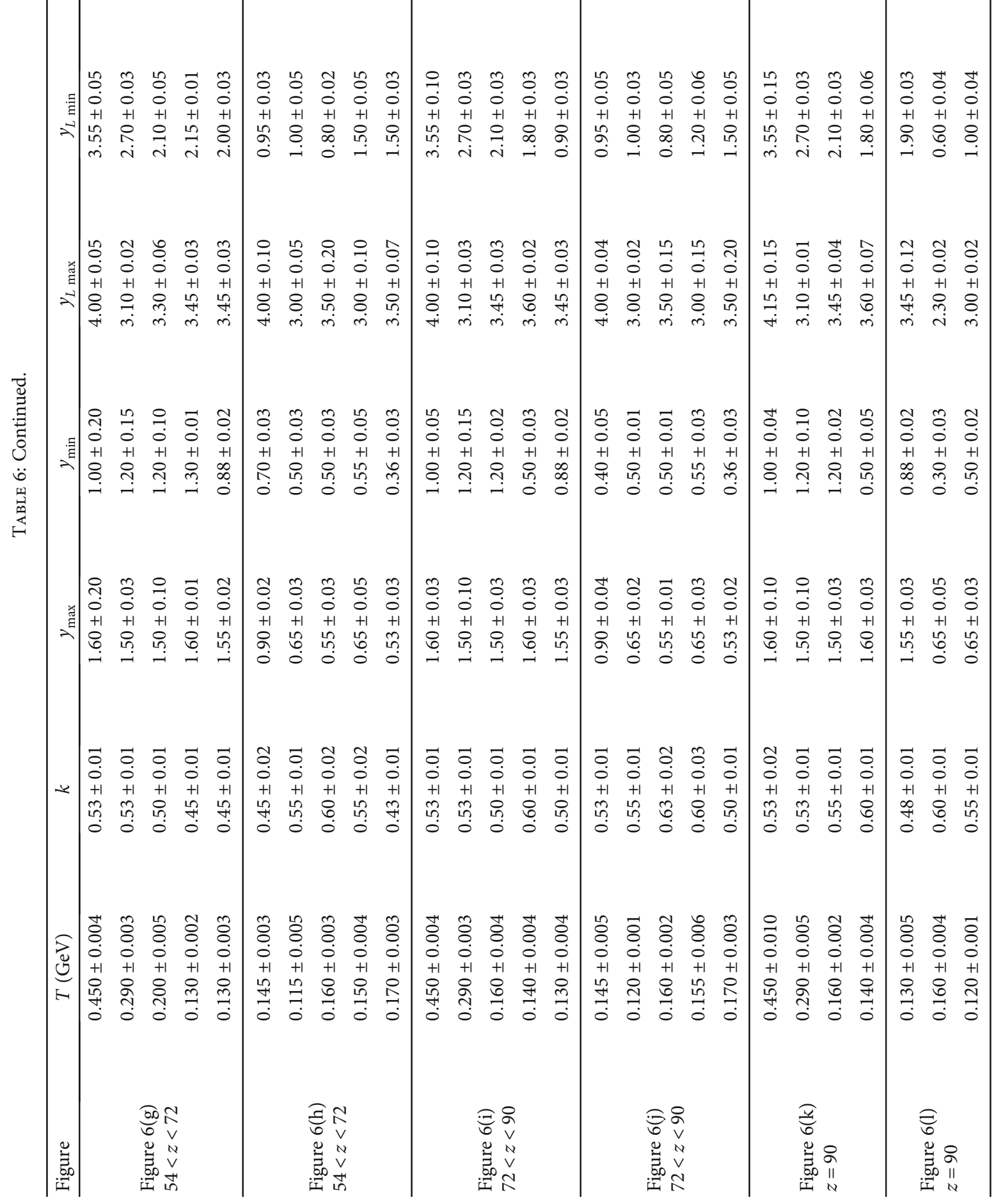




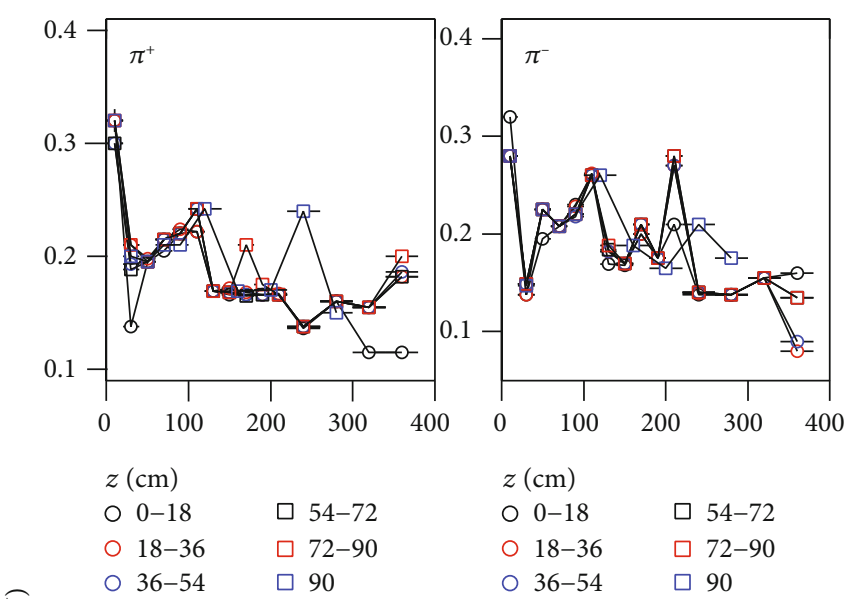

(a)

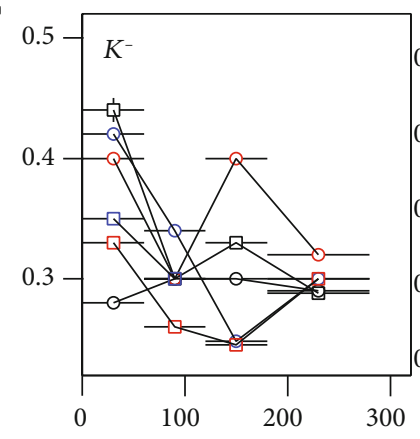

$\begin{array}{ll}z(\mathrm{~cm}) & \\ \circ 0-18 & \square 54-72 \\ \circ 18-36 & \square 72-90 \\ \circ 36-54 & \square 90\end{array}$

(d)

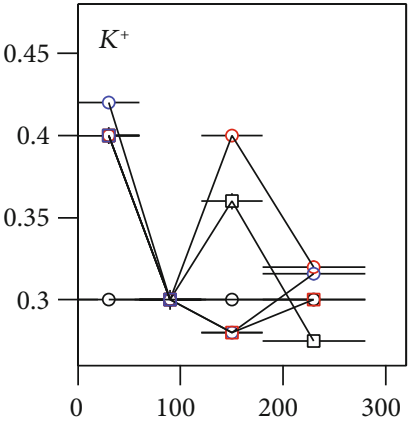

$z(\mathrm{~cm})$

○ $0-18$

○ $18-36$

$\square$ 54-72

$\square 72-90$

○ 36-54 $\square 90$

(c)

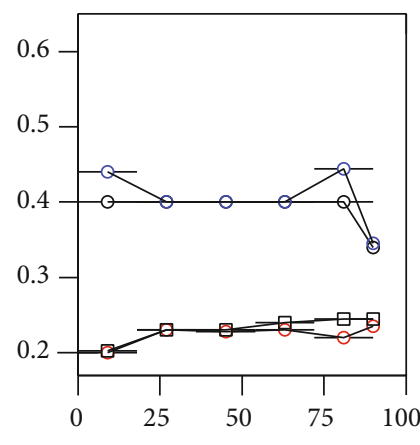

$\theta$ (mrad)

$\pi^{+}$○ 20-40

○ $100-140$

$\pi^{-} \circ 20-40$

$\square$ 100-140

(f)

Figure 7: Dependence of $T$ on (a-e) $\theta$, which are extracted from the data samples within different $z$ ranges for $\pi^{+}, \pi^{-}, K^{+}, K^{-}$, and $p$, respectively, and on (f) $z$, which are extracted from the data samples within different $\theta$ ranges for $\pi^{+}$and $\pi^{-}$.

C collisions at $31 \mathrm{GeV} / \mathrm{c}$ in the laboratory reference frame, respectively, where $N_{\text {pot }}$ denotes the number of protons on target and $n$ denotes the number of particles. Panels $(\mathrm{a}-\mathrm{c})$, $(\mathrm{d}-\mathrm{f}),(\mathrm{g}-\mathrm{i}),(\mathrm{j}-\mathrm{l}),(\mathrm{m}-\mathrm{o})$, and $(\mathrm{p}-\mathrm{q})$ represent the spectra for $z=0-18,18-36,36-54,54-72,72-90$, and $90 \mathrm{~cm}$, respectively. For clarity, spectra in different $\theta$ ranges are scaled by adding different numbers (marked in the panels) represented by different symbols, which are the experimental data measured by the NA61/SHINE Collaboration [32]. The curves are our results fitted by the multisource thermal model using to Equation (1) and the Monte Carlo method. The values of free parameters $\left(T, y_{\max }\right.$, and $\left.y_{\min }\right)$, normalization constant $\left(N_{0}\right), \chi^{2}$, and number of degree of freedom (ndof) corresponding to the fits for the spectra of $\pi^{+}$and $\pi^{-}$are listed in Tables 1 and 2, respectively. In two cases, ndof in the fittings are negative which appear in the tables with "-" signs and the corresponding curves are for eye guiding only. One can see that the theoretical model results are approximately in agreement with the NA61/SHINE experimental data of $\pi^{+}$and $\pi^{-}$.
Figure 3 presents the momentum spectra of $(a, b) \pi^{+}$and (c, d) $\pi^{-}$in (a-c) $\theta=20-40 \mathrm{mrad}$ and (b-d) $\theta=100-$ $140 \mathrm{mrad}$ in $\operatorname{six} z$ ranges with different scaled amounts shown in the panels. The symbols represent the experimental data [32]. The curves are our results fitted by the model. The values of $T, y_{\max }, y_{\min }, N_{0}, \chi^{2}$, and ndof corresponding to the fits for the spectra of $\pi^{+}$and $\pi^{-}$are listed in Table 3 . One can see again that the theoretical model results are approximately in agreement with the experimental data of $\pi^{+}$and $\pi^{-}$.

Similar to Figures 1 and 2, Figures 4 and 5 show the momentum spectra of positively and negatively charged kaons $\left(K^{+}\right.$and $\left.K^{-}\right)$produced in $p$-C collisions at $31 \mathrm{GeV} / c$, respectively. Panels (a-f) represent the spectra for $z=0-18$, $18-36,36-54,54-72,72-90$, and $90 \mathrm{~cm}$, respectively. The values of $T, y_{\max }, y_{\min }, N_{0}, \chi^{2}$, and ndof corresponding to the fits for the spectra of $K^{+}$and $K^{-}$are listed in Tables 4 and 5 , respectively. One can see that the theoretical model results are approximately in agreement with the experimental data of $K^{+}$and $K^{-}$. 


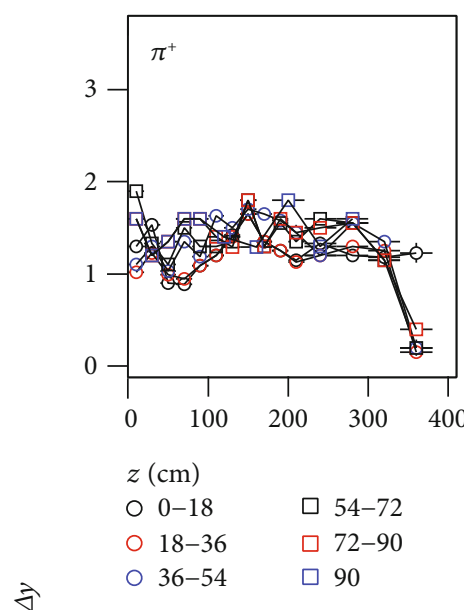

(a)
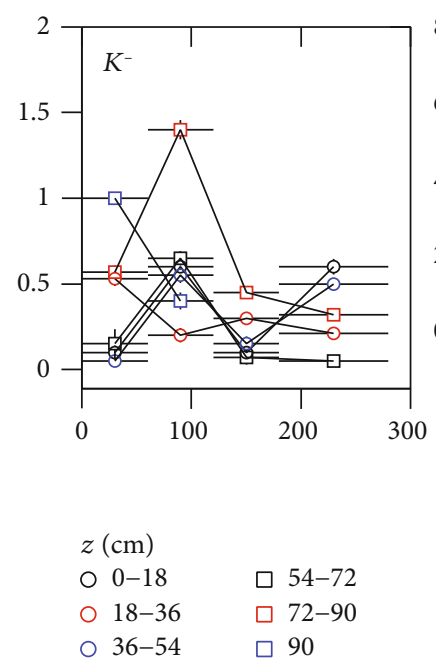

(d)

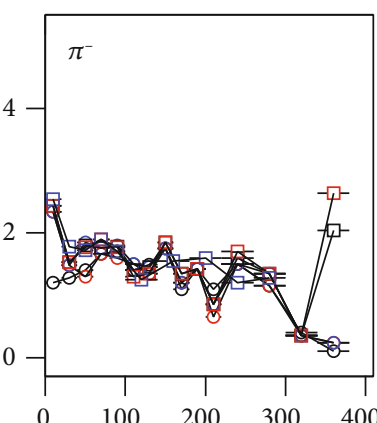

$z(\mathrm{~cm})$

○ $0-18$

○ $18-36$

○ $36-54$

$\square 54-72$

$\square 72-90$

$\square 90$

(b)

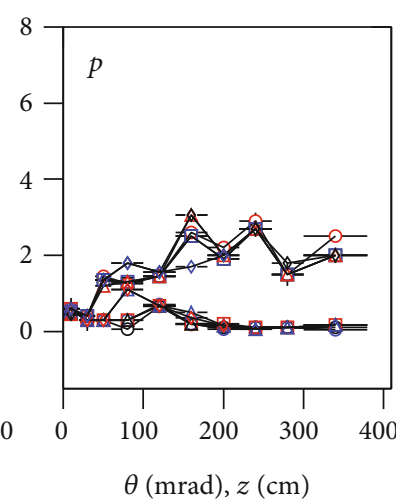

$$
\begin{array}{ll}
z(\mathrm{~cm}) & \\
\text { ○० } 0-18 & \Delta \Delta 54-72 \\
\text { ㅁ } 18-36 & \Delta \diamond 72-90 \\
\text { ㅁ 36-54 } & \diamond \diamond 90
\end{array}
$$

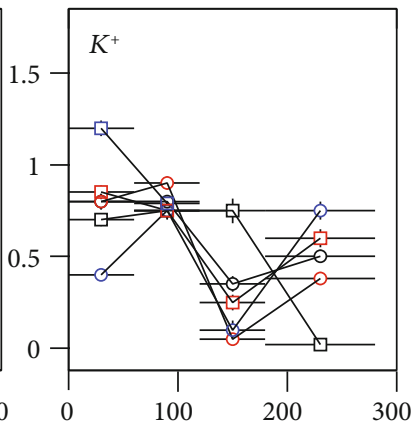

$z(\mathrm{~cm})$

$$
\begin{aligned}
& \text { ○ } 0-18 \\
& \text { 54-72 }
\end{aligned}
$$

$\square 90$

(c)

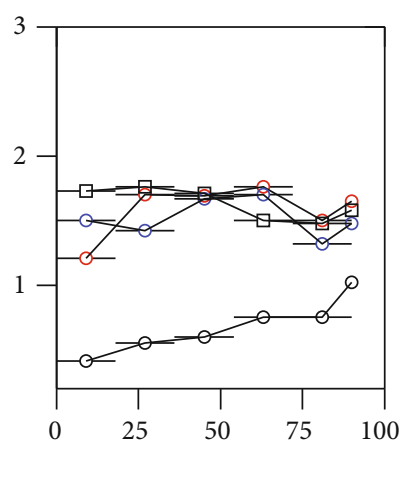

$$
\begin{aligned}
& \theta(\mathrm{mrad}) \\
& \pi^{+} \bigcirc 20-40 \\
& \bigcirc 100-140 \\
& \pi^{-} \bigcirc 20-40 \\
& \square 100-140
\end{aligned}
$$

(e)

FiguRE 8: Same as Figure 7 but showing the dependence of $\Delta y$. Large $\Delta y\left(=y_{L \max }-y_{L \text { min }}>1\right)$ in panel (e) represents mainly the rapidity shifts for leading protons.

Similar to Figure 1, Figure 6 shows the momentum spectra of $p$ emitted in $p$-C collisions at $31 \mathrm{GeV} / c$. Panels $(a, b),(c, d),(e, f),(g, h),(i, j)$, and $(k, l)$ represent the spectra for $z=0-18,18-36,36-54,54-72,72-90$, and $90 \mathrm{~cm}$, respectively. The values of $T, k, y_{\max }, y_{\min }, y_{L \max }$, $y_{L \text { min }}, N_{0}, \chi^{2}$, and ndof corresponding to the fits for the spectra are listed in Table 6. In a few cases, ndof are negative which appear in the table in terms of "-" and the corresponding curves are just for eye guiding only. It should be noted that the contributions of leading protons have to be considered in the spectra. One can see that the theoretical model results are approximately in agreement with the experimental data.

We notice from Tables 1-6 that different $T$ for a range of $z$ and its dependence with $\theta$ or $y$ are observed, but the development of the model in our previous work [42] concludes that $T$ is independent of $y$. We would like to explain here that this paper treats $T$ as a differential function of $\theta$ or $y$, which is more detailed, while our previous work treats $T$ as an integral or mean quantity over $y$. As for which case should be used, it depends on the experimental data.

We now analyze the dependence of free parameters on $\theta$ and $z$. Figures 7 and 8 show, respectively, the dependence of $T$ and $\Delta y\left(=y_{\max }-y_{\min }\right)$ on (a-e) $\theta$, which is extracted from the data samples within different $z$ ranges for $\pi^{+}, \pi^{-}, K^{+}, K^{-}$, and $p$, respectively, and on (f) $z$, which are extracted from the data samples within different $\theta$ ranges for $\pi^{+}$and $\pi^{-}$, where we use $\Delta y$ to denote the difference between $y_{\max }$ and $y_{\min }$ to avoid trivialness in using both $y_{\max }$ and $y_{\min }$. In particular, in Figure 8(e), the results with $\Delta y>1$ are mainly for leading protons and obtained by $y_{L \max }-y_{L \min }$. One can see that, for $\pi^{ \pm}$and $K^{ \pm}, T$ and $\Delta y$ decrease slightly with the increase of $\theta$ and do not change obviously with the increase of $z$. The obtained $T(\Delta y)$ values for negative and positive pions or kaons seem to be very similar as we expect. The data for the antiproton $(\bar{p})$ are not available in Ref. [32], which forbids in making a comparison for $p$ and $\bar{p}$ in this paper. In fact, the 


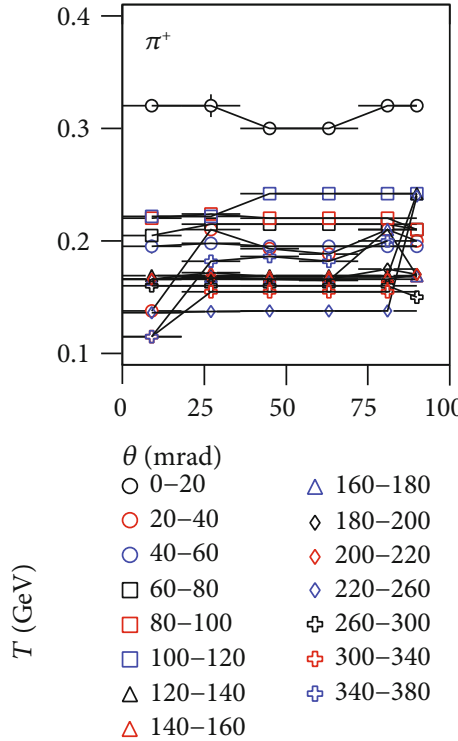

(a)

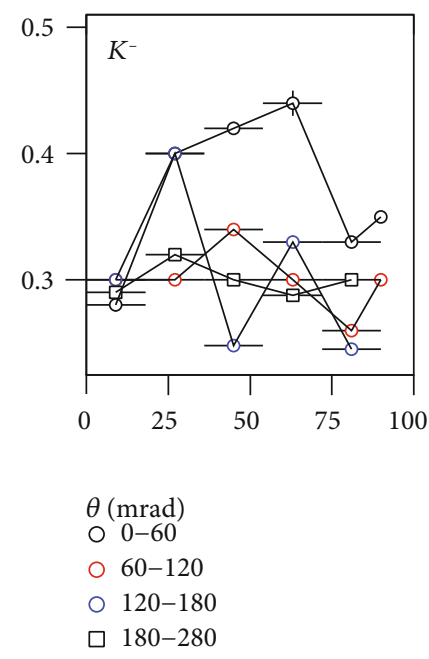

(d)

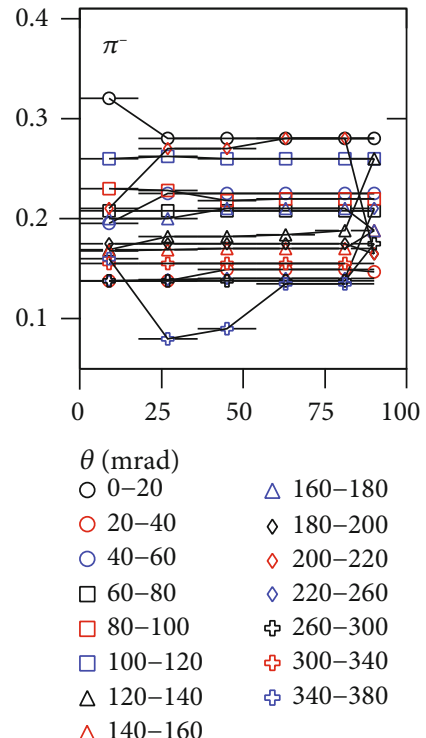

(b)
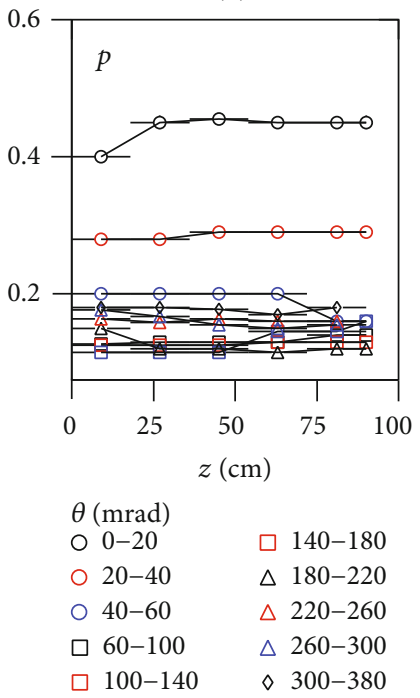

(e)

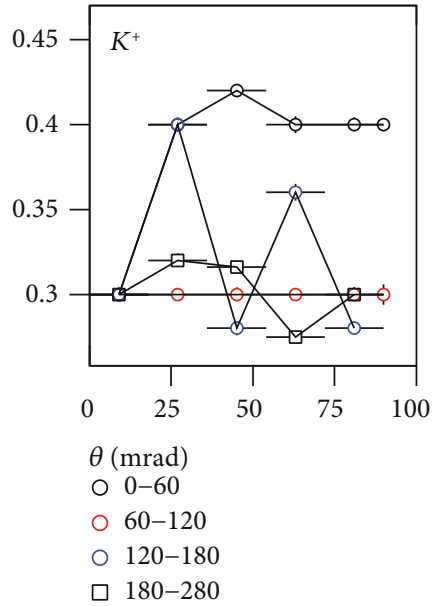

(c)

Figure 9: Dependence of $T$ on $z$, which are extracted from the data samples within different $\theta$ ranges for (a) $\pi^{+}$, (b) $\pi^{-}$, (c) $K^{+}$, (d) $K^{-}$, and (e) $p$.

situation for $p$ is more complex due to the effect of leading protons.

The dependence of $T$ and $\Delta y$ on $\theta$ for the productions of $\pi^{ \pm}$and $K^{ \pm}$can be explained by the effect of cascade collisions in the target and by the nuclear stopping of the target. The cascade collisions can cause larger $\theta$ and more energy loss and then lower $T$. The nuclear stopping can cause smaller $\Delta y$. Combining with cascade collisions and nuclear stopping, one can obtain low $T$ and small $\Delta y$ at large $\theta$ for the productions of $\pi^{ \pm}$and $K^{ \pm}$. Because of the effect of leading particles, the situation for the emissions of $p$ is more complex, which shows different trends from those of $\pi^{ \pm}$and $K^{ \pm}$. Meanwhile, the flow effect can cause larger $T$, which is related to a more complex mechanism.

The dependence of $T$ and $\Delta y$ on $z$, which are extracted from the data samples within different $\theta$ ranges for (a) $\pi^{+}$, (b) $\pi^{-}$, (c) $K^{+}$, (d) $K^{-}$, and (e) $p$, are given in Figures 9 and
10 , respectively. In particular, large $\Delta y\left(=y_{L \max }-y_{L \text { min }}>1\right)$ in Figure 10(e) represent mainly the rapidity shifts of leading protons. In principle, there is no obvious increase or decrease in $T$ and $\Delta y$ with the increase of $z$, but there are some statistical fluctuations in a few cases. This result is natural due to the fact that $z$ is not the main factor in a $90 \mathrm{~cm}$ long graphite target. It is expected that $T$ and $\Delta y$ will decrease with the increase of $z$ in a very long graphite target in which the energy loss of the beam protons has to be considered. The NA61/SHINE experimental data analyzed in this paper are not obtained from a long graphite target, and hence, it is not necessary to consider the energy loss of the beam protons.

Figure 11 displays the dependence of fraction $k$ of nonleading protons on (a) $\theta$ and (b) $z$, which are extracted from the data samples within different $z$ and $\theta$ ranges, respectively. One can see that there is no obvious change in the 


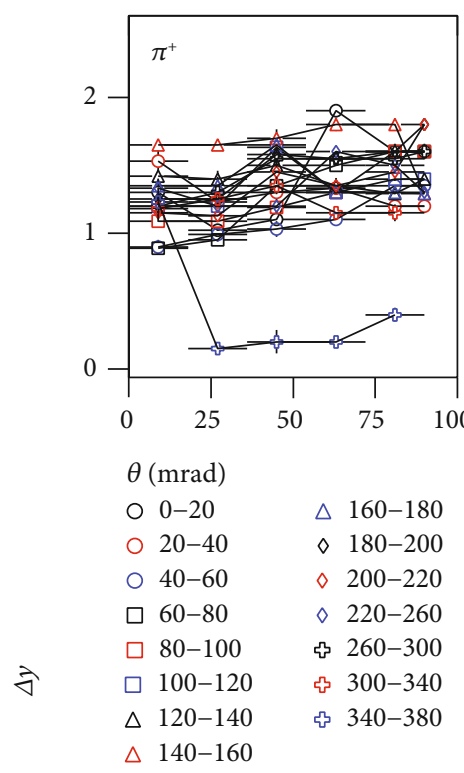

(a)

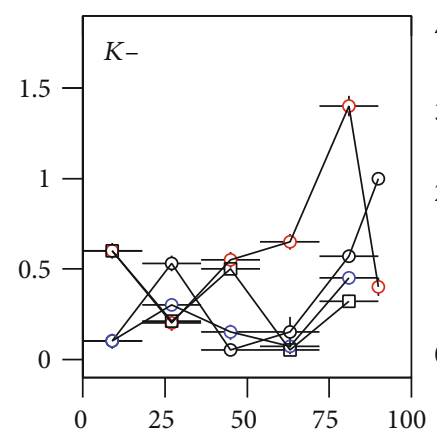

$\theta(\mathrm{mrad})$

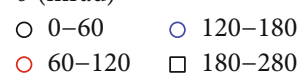

(d)

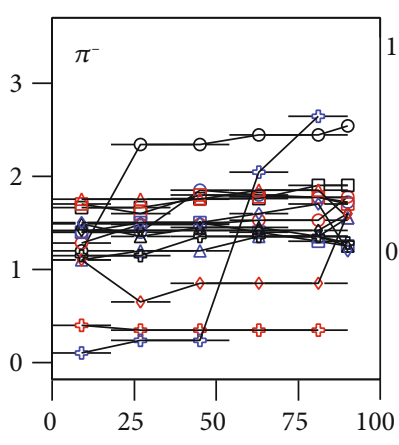

$\theta(\mathrm{mrad})$

○ $0-20$

○ $20-40$

$\triangle 160-180$

○ $40-60$

60-80

$\square$ 80-100

$\square 100-120$

$\triangle 120-140$

$\triangle 140-160$

(b)

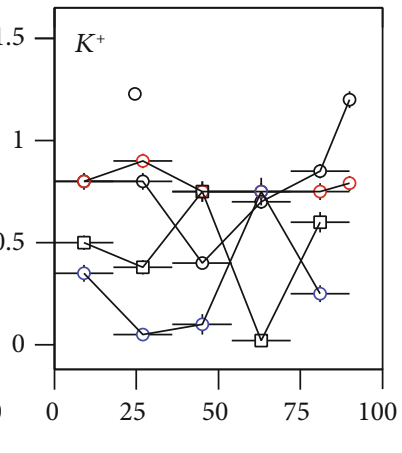

$\theta(\mathrm{mrad})$

○ $0-60 \quad$ O $120-180$

○ $60-120$ 口 180-280

$180-200$

$\diamond 200-220$

$\diamond 220-260$

๘ 260-300

ङ 300-340

Æ 340-380

(c)

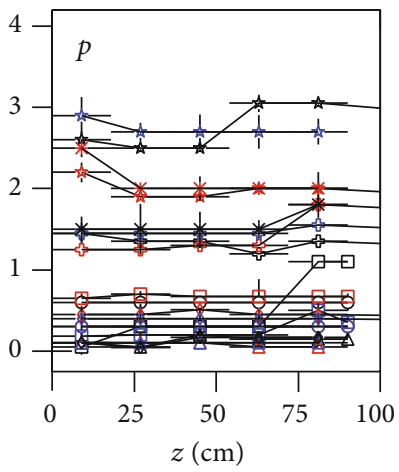

$$
\begin{aligned}
& \theta \text { (mrad) }
\end{aligned}
$$

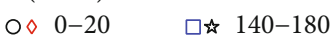

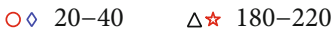

$$
\begin{aligned}
& \text { О‡ 40-60 } \Delta \text { म 220-260 } \\
& \text { 口ई 60-100 } \triangle \text { क 260-300 } \\
& \text { 口ヶ 100-140 ه* 300-380 }
\end{aligned}
$$

(e)

Figure 10: Same as Figure 9 but showing the dependence of $\Delta y$. Large $\Delta y\left(=y_{L \max }-y_{L \text { min }}>1\right)$ in panel (e) represents mainly the rapidity shifts for leading protons.

dependence of $k$ on $\theta$, but some statistical fluctuations. There is a slight increase in the dependence of $k$ on $z$ with the increase of $z$, which can be explained by more energy loss of the beam protons at larger $z$. This energy loss is small in a not too large $z$ range, which does not affect obviously other free parameters such as $T$ and $\Delta y$ due to their less sensitivity at the energy in the $z$ range considered in this paper. It is natural that the larger (fewer) fraction $k(1-k)$ of protons appears as nonleading (leading) particles at lower energy or larger $z$. Indeed, the fraction is mainly determined by the collision energy, and the leading protons are considerable at the SPS. In fact, the leading protons are those that existed in the projectile with high momentum and small emission angle, but not the produced protons. With the increase of collision energy up to dozens of $\mathrm{GeV}$ and above at which meson-dominated final states appear [43], $k$ will increase due to the increase of accompanied produced protons. With the decrease of collision energy down to several $\mathrm{GeV}$ and below at which baryondominated final states appear [43], $k$ will also increase due to the increase of target stopping which causes the decrease of leading protons.

Figures 12 and 13 show, respectively, the dependence of average $p_{T}\left(\left\langle p_{T}\right\rangle\right)$ and $T_{i}$ on (a-e) $\theta$, which are extracted from the data samples within different $z$ ranges for $\pi^{+}, \pi^{-}, K^{+}, K^{-}$, and $p$, respectively, and on (f) $z$, which are extracted from the data samples within different $\theta$ ranges for $\pi^{+}$and $\pi^{-}$, where $T_{i}$ denotes the initial quasitemperature which is given by 


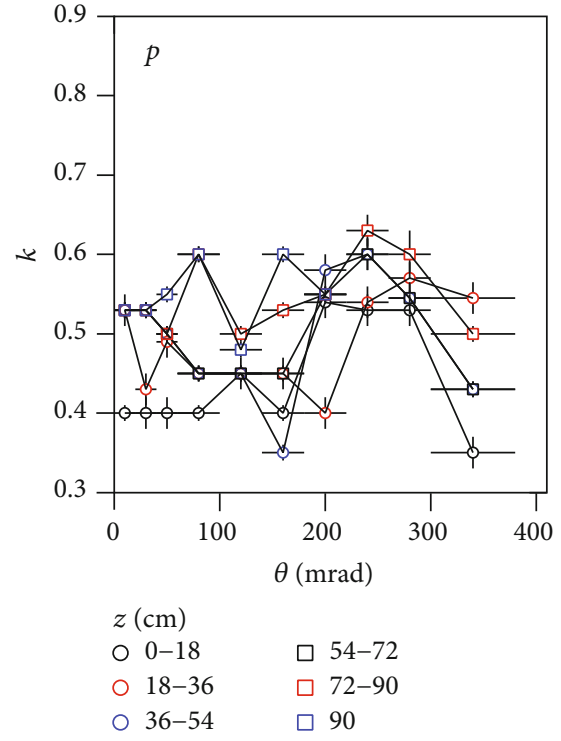

(a)

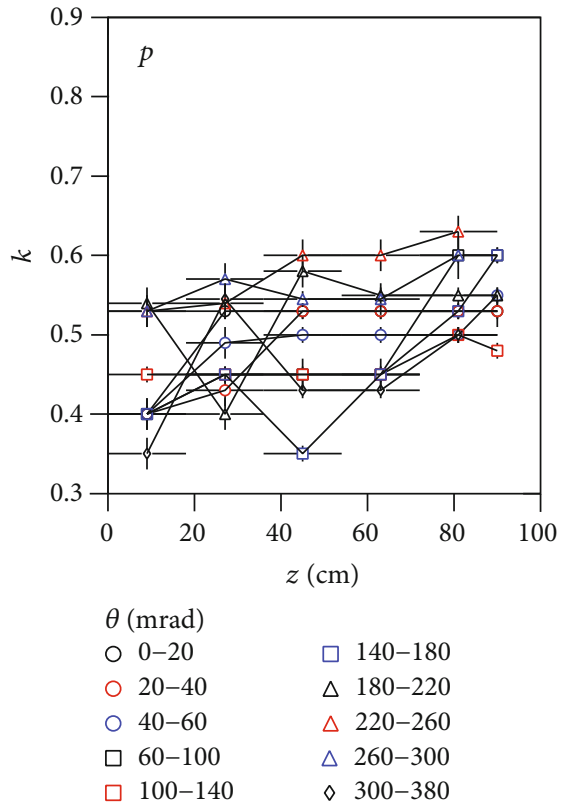

(b)

Figure 11: Dependence of $k$ on (a) $\theta$ and (b) $z$, which are extracted from the data samples within different $z$ and $\theta$ ranges, respectively.

the root-mean-square $p_{T}\left(\sqrt{\left\langle p_{T}^{2}\right\rangle}\right)$ over $2\left(\sqrt{\left\langle p_{T}^{2}\right\rangle / 2}\right)$ according to the color string percolation model [44-46]. It should be noted that $\sqrt{\left\langle p_{T}^{2}\right\rangle / 2}$ in Refs. [44-46] is regarded as the initial temperature. In that model, there are free parameters associated with the medium created in a high-energy collision, which is not the case for this paper at low energy. So we call $\sqrt{\left\langle p_{T}^{2}\right\rangle / 2}$ the initial quasitemperature in this paper. The dependence of $\left\langle p_{T}\right\rangle$ and $T_{i}$ on $z$ are presented in Figures 14 and 15, respectively, which are extracted from the data samples within different $\theta$ ranges. One can see that, for pions and kaons, there are increases in $\left\langle p_{T}\right\rangle$ and $T_{i}$ when $\theta$ increases. The situation is complex for protons due to the effect of leading protons which have high momenta and result in high $\left\langle p_{T}\right\rangle$ and $T_{i}$ at small $\theta$. The produced protons which are nonleading should have a similar trend in $\left\langle p_{T}\right\rangle$ and $T_{i}$ as those for pions and kaons. As a combination, the final protons are the sum of leading and produced protons. There is no obvious change in $\left\langle p_{T}\right\rangle$ and $T_{i}$ when $z$ increases due to not too large energy loss in a $90 \mathrm{~cm}$ long graphite target.

We would like to point out that there are different definitions [47] for leading particles in experiments. There are at least four production mechanisms $[48,49]$ for leading protons in electron-induced deep-inelastic scattering on protons. Among these mechanisms, at HERA energy, diffractive deep-inelastic scattering $[50,51]$ in which $72 \%$ of leading protons have momentum being larger than $0.9 p_{\mathrm{Lab}}$ occupy about $26 \%$ [48] of leading protons, which are not enough to cover all leading protons. In particular, for leading protons with momenta being $(0.5-0.98) p_{\mathrm{Lab}}$, a large fraction $(77 \%)$ comes from nondiffractive deep-inelastic scatterings. In proton-proton and proton-nucleus collisions at the considered energy level of this paper, the fraction of the diffractive process is about 20\% [52] in inelastic events, which is only half of the fraction of leading protons. Even in nucleus-nucleus collisions, the effect of leading protons in the forward rapidity region is also obvious [42, 5355], which also reflects in a high momentum region and is not only from diffractive process.

Naturally, there are other additional arguments to explain the behavior of Figures 12 and 13 for the proton case. In fact, there are multiple or cascade secondary scatterings among produced particles and target nucleons. As low mass particles, the emission angles of pions and kaons increase obviously after multiple scatterings. This results in large $\left\langle p_{T}\right\rangle$ and $T_{i}$ due to large $\theta$ for pions and kaons. Contrary to this, the emission angles of protons increase in smaller amount after multiple scatterings due to higher mass of protons compared to pions and kaons. This results in small $\left\langle p_{T}\right\rangle$ and $T_{i}$ due to small $\theta$ for protons. However, nonnegligible leading protons which have high momenta and smaller angles do not experience much multiple scatterings, which renders large $\left\langle p_{T}\right\rangle$ and $T_{i}$ at small $\theta$. As a competitive result, protons present a different case from pions and kaons.

One can see naturally the coincident trend for $\left\langle p_{T}\right\rangle$ and $T_{i}$ in different $\theta$ and $z$ ranges. Due to the flow effect not being excluded, the trend of $T$ is inconsistent with that of $T_{i}$. As an all-around result, the effects of transverse and longitudinal flows are complex. The flow effect can obviously affect $T$, which is model dependent. The flow effect also affects $\left\langle p_{T}\right\rangle$ and $T_{i}$ which are also model dependent. Therefore, we mention here that $T$ is not a "real" temperature, but the effective temperature. In our opinion, the temperature and flow velocity should be independent of models, which is usually not the case more often, as some formalism is used 


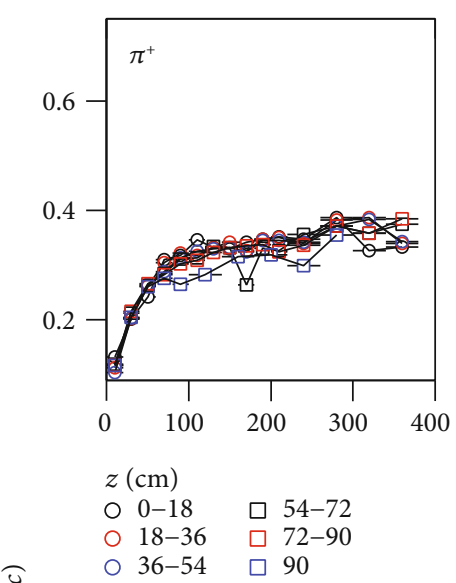

(a)

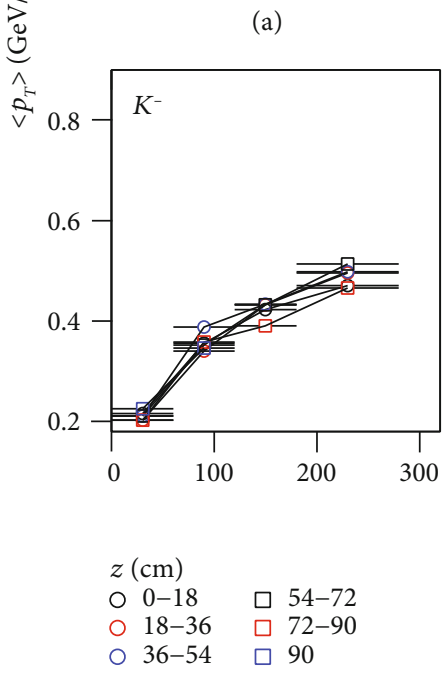

(d)

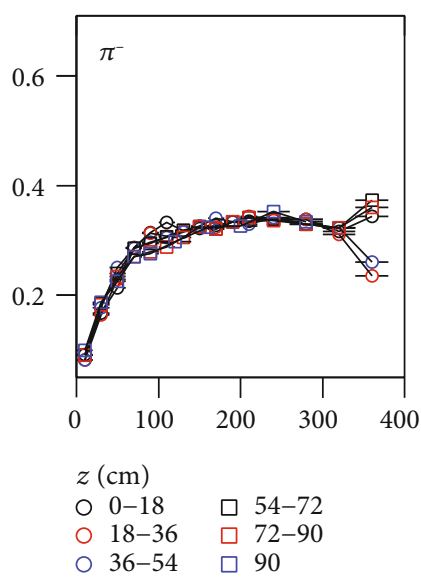

(b)

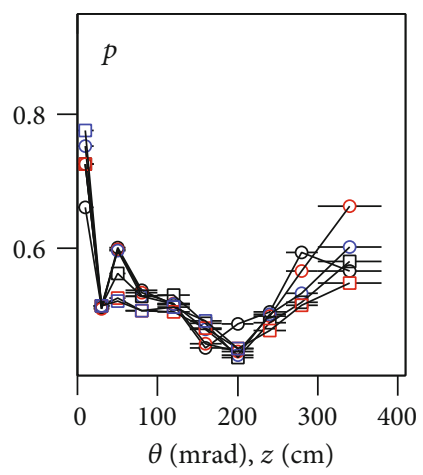

$z(\mathrm{~cm})$

○ $0-18 \square$ $54-72$

$\circ 18-36 \square 72-90$

○ $36-54 \square 90$

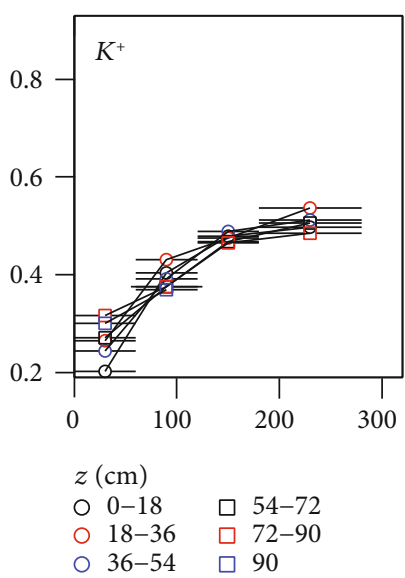

(c)

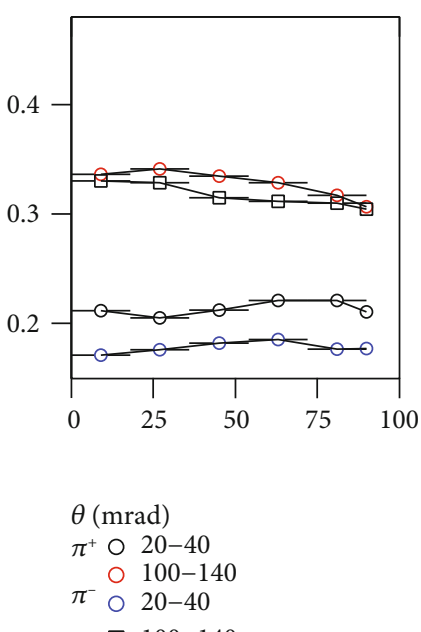

(f)

Figure 12: Dependence of $\left\langle p_{T}\right\rangle$ on (a-e) $\theta$, which are extracted from the data samples within different $z$ ranges for $\pi^{+}, \pi^{-}, K^{+}, K^{-}$, and $p$, respectively, and on (f) $z$, which are extracted from the data samples within different $\theta$ ranges for $\pi^{+}$and $\pi^{-}$.

to extract the radial flow and the real/thermal temperature, which estimate the real temperature of the system being dependent of models.

The experimental data cannot be clearly distinguished into two parts: One part is the contribution of thermal motion, which reflects the "real" temperature at the kinetic freeze-out. The other part is the contribution of the collective flow. The current blast-wave model $[34,35]$ treats the thermal motion and flow effect by using the kinetic freeze-out temperature and transverse flow velocity, respectively. After fitting the spectra with ndof $>1$ and using $p_{T}$ coverage as widely as possible $\left(p_{T}=0-3 \mathrm{GeV} / c\right)$, our study using the blast-wave model with flow profile parameter being 2 can obtain similar fit results as the curves in Figures 1-6. To protrude the fit results of the thermal model, the fit results of the blast-wave model are not displayed in these figures. The relation between $T_{0}$ and $\beta_{T}$ for different cases from the spectra of positive particles is plotted in Figure 16, where the circles, squares, and triangles represent the results from $\pi^{+}, K^{+}$, and $p$ spectra, respectively. One can see considerable flow-like effect in $p$-C collisions at $31 \mathrm{GeV} / c$, which shows a positive correlation between $T_{0}$ and $\beta_{T}$. The kinetic freezeout temperature $T_{0}$ is about from 0.080 to $0.135 \mathrm{GeV}$. The corresponding transverse flow velocity $\beta_{T}$ is about from 0.21 to $0.42 c$. Massive particles such as $p$ correspond to larger $T_{0}$ and smaller $\beta_{T}$ comparing to $\pi^{+}$at the same or similar $\theta$, which is in agreement with hydrodynamictype behavior. The flow-like effect observed in this work is slightly less than the flow velocity $(0.3 c$ in peripheral and $0.5 c$ in central gold-gold collisions) obtained from the yield ratio of $p / \pi$ in a simple afterburner model [56]. The difference is due to the fact that a lower energy small system with minimum-bias sample is studied in this paper. In some cases, the results on kinetic freeze-out temperature or transverse flow velocity obtained from different models are not always harmonious [36, 37].

It should be noted that there is entanglement in determining $T_{0}$ and $\beta_{T}$. For a given $p_{T}$ spectrum, $T_{0}$ and $\beta_{T}$ are negatively correlated, which means an increase in $T$ should result in a decrease of $\beta_{T}$. But for a set of $p_{T}$ spectra, after determining $T_{0}$ and $\beta_{T}$ for each $p_{T}$ spectrum, the correlation between $T_{0}$ and $\beta_{T}$ is possibly positive or negative, which 


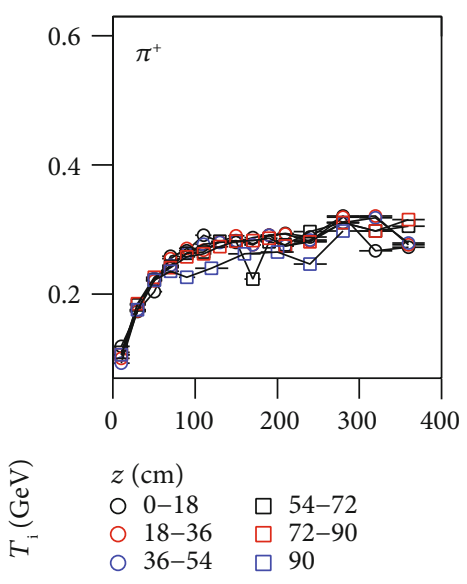

(a)

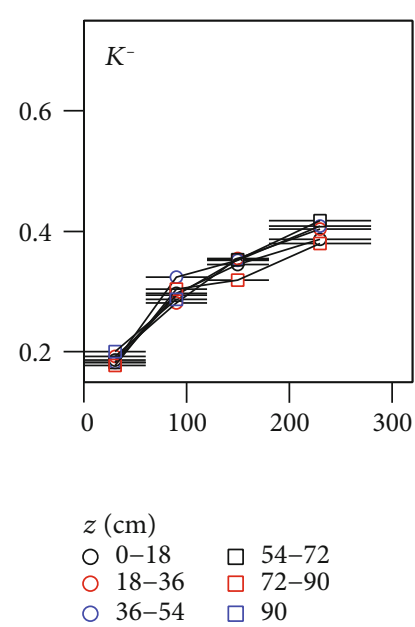

(d)
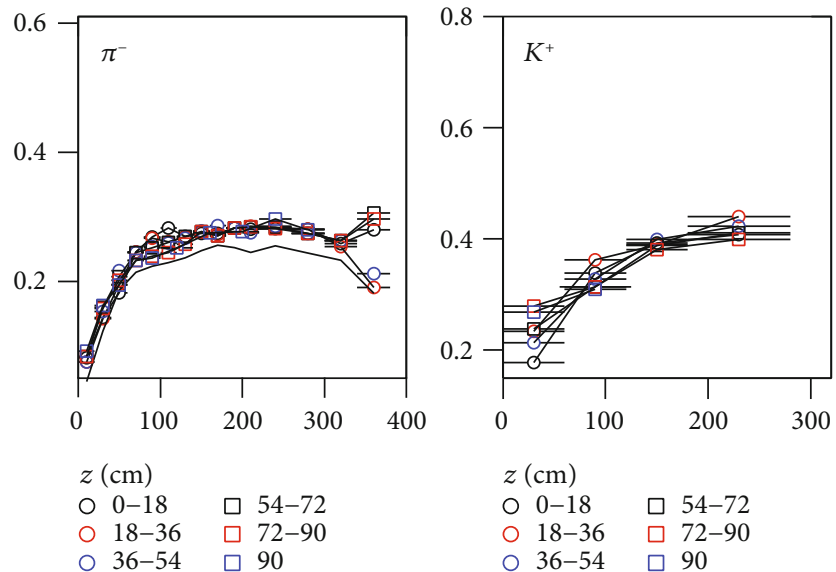

(c)
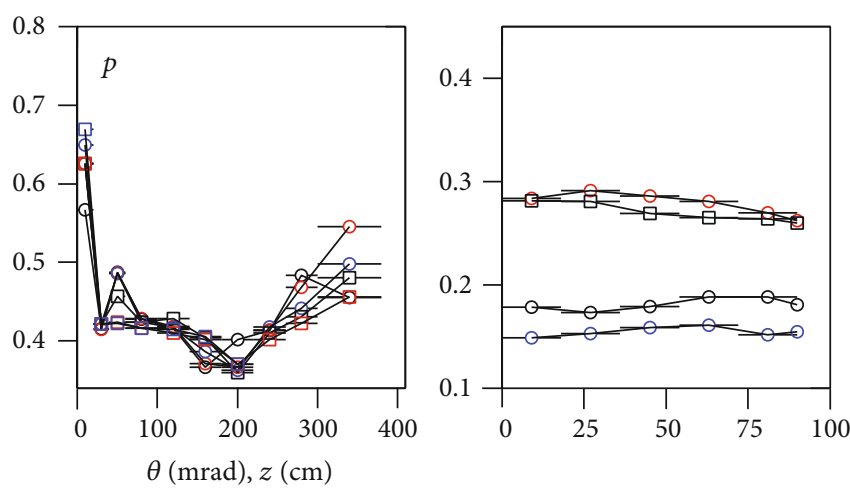

$$
\begin{array}{ll}
z(\mathrm{~cm}) & \\
\circ 0-18 & \square 54-72 \\
\circ 18-36 & \square 72-90 \\
\circ 36-54 & \square 90
\end{array}
$$

(e)

FIgURE 13: Same as Figure 12 but showing the dependence of $T_{i}$.

depends on the choices of flow profile function and $p_{T}$ coverage. If the correlation is negative, one may increase $T_{0}$ and decrease $\beta_{T}$ by changing the flow profile function and $p_{T}$ coverage and obtain possibly positive correlation. If the correlation is positive, one may decrease $T_{0}$ and increase $\beta_{T}$ by changing the flow profile function and $p_{T}$ coverage and obtain possibly negative correlation. Unlike experimental papers, where one finds a single $T_{0}$ and a common $\beta_{T}$ by fitting the blast-wave model to the bulk part of the $p_{T}$ spectra (in a very narrow coverage which is particle dependent and much less than $3 \mathrm{GeV} / c$ ) by performing a simultaneous fitting to the identified particle spectra using a changeable $n_{0}$ (from 0 to 4.3) [57], here we have considered a differential freeze-out scenario and have restricted uniformly the fitting up to $3 \mathrm{GeV} / c$ for different particles and have used always $n_{0}=2$. The value of $T_{0}\left(\beta_{T}\right)$ in positive correlation is larger (less) than that in negative correlation. Positive correlation means high excitation and quick expansion, while negative correlation means longer lifetime (lower excitation) and quicker expansion. In our opinion, although both positive and negative correlations are available, one needs other methods to check which one is suitable. In fact, the positive correlation in Figure 16 is in agreement with the alternative method used in our previous works $[36,37]$.

We would rather like to use $\left\langle p_{T}\right\rangle$ directly in the determination of kinetic freeze-out temperature and transverse flow velocity. For example, the contribution of one participant in each binary collision in the Erlang distribution is $\left\langle p_{T}\right\rangle / 2$ which is regarded as effective temperature [58] contributed by the thermal motion and flow effect. We could assume the contribution fraction of the thermal motion to be $k_{0}$. Then, the kinetic freeze-out temperature is $k_{0}\left\langle p_{T}\right\rangle / 2$, and the transverse flow velocity is $\left(1-k_{0}\right)\left\langle p_{T}\right\rangle / 2 m_{0} \bar{\gamma}$, where $\bar{\gamma}$ is the mean Lorentz factor of the considered particles in the rest frame of emission source. If we take $k_{0} \approx 0.3$ and at large $\theta$, the obtained kinetic freeze-out temperatures $(0.05 \mathrm{GeV}$ for pion emission and $0.10 \mathrm{GeV}$ for proton emission) are in agreement with those from the blast-wave model $[34,35]$ and transverse flow velocities $(0.2 c$ for pion emission and $0.1 c$ for proton emission) are qualitatively in agreement with those from the blast-wave model $[34,35]$ and the afterburner model [50]. The treatment of $\left\langle p_{T}\right\rangle / 2$ is also model dependent 

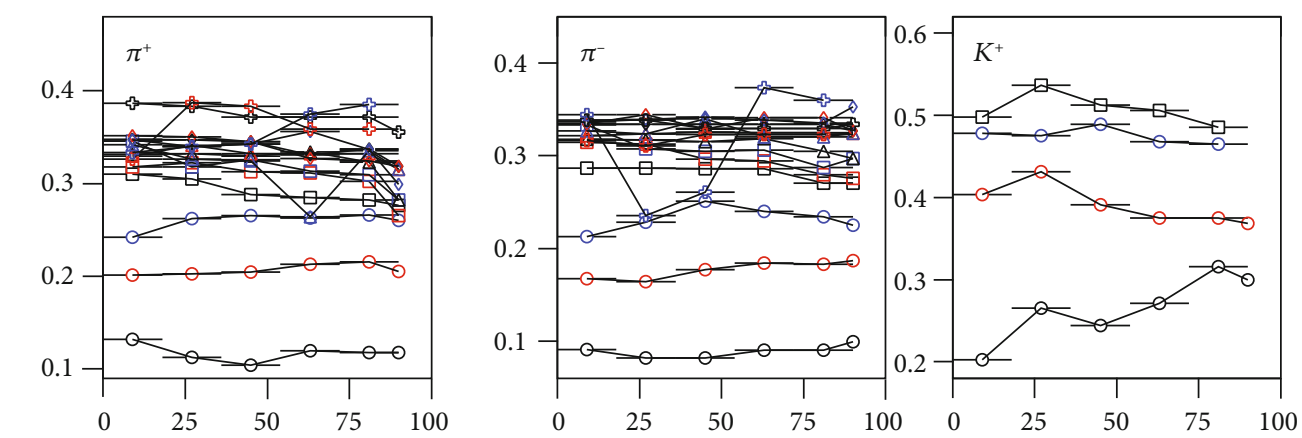

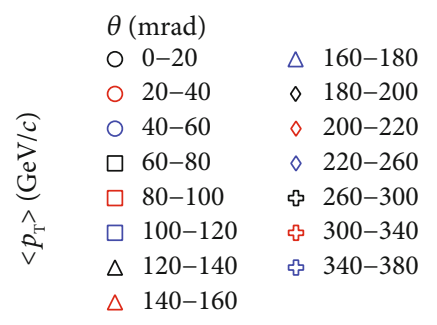

(a)

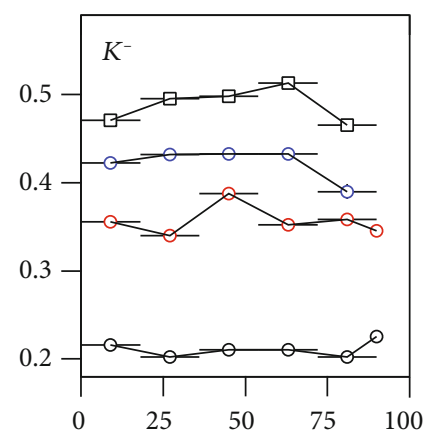

$\theta(\mathrm{mrad})$

○ $0-60 \quad \circ \quad 120-180$

○ $60-120 \quad \square \quad 180-280$

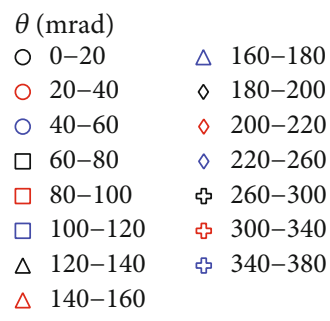

(b)

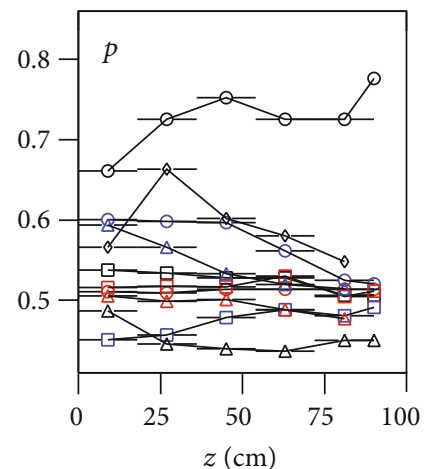

$\theta(\mathrm{mrad})$

$$
\begin{array}{ll}
\circ 0-20 & \square 140-180 \\
\circ 20-40 & \triangle 180-220 \\
\circ 40-60 & \triangle 220-260 \\
\square 60-100 & \triangle 260-300 \\
\square 100-140 & \triangle 300-380
\end{array}
$$

(e)

\section{$\theta(\mathrm{mrad})$ \\ ○ $0-60 \quad$ O $120-180$}

○ $60-120 \square 180-280$

(d)

Figure 14: Dependence of $\left\langle p_{T}\right\rangle$ on $z$, which are extracted from the data samples within different $\theta$ ranges for (a) $\pi^{+}$, (b) $\pi^{-}$, (c) $K^{+}$, (d) $K^{-}$, and (e) $p$.

and in agreement with hydrodynamic-type behavior. In addition, larger $\left\langle p_{T}\right\rangle / 2$ results in larger $T_{0}$ and $\beta_{T}$, which shows positive correlation between $T_{0}$ and $\beta_{T}$. The positive correlation in Figure 16 is also in agreement with the treatment of $\left\langle p_{T}\right\rangle / 2$.

Before the summary and conclusions, we would like to point out that the kinetic freeze-out temperature and transverse flow velocity obtained in this paper are mass dependent, which renders a scenario for multiple kinetic freezeout (differential freeze-out) [59]. The afterburner model [56] uses a mass-independent flow velocity, which renders a scenario for single kinetic freeze-out. There are arguments on the kinetic freeze-out scenario, which is beyond the focus of this paper, so we shall not discuss it anymore. In addition, it should be noted that in the absence of a required number of experimental data points, the fittings using the current model in few cases yield negative $\chi^{2}$ /ndof, making the description unphysical, though the corresponding curves could be used as eye guiding only.

\section{Summary and Conclusions}

We summarize here our main observations and conclusions:

(a) The momentum spectra of $\pi^{+}, \pi^{-}, K^{+}, K^{-}$, and $p$ produced in $p-\mathrm{C}$ collisions at $31 \mathrm{GeV} / c$ are analyzed in the framework of a multisource thermal model by using the Boltzmann distribution and Monte Carlo method. The results are approximately in agreement with the experimental data in various 


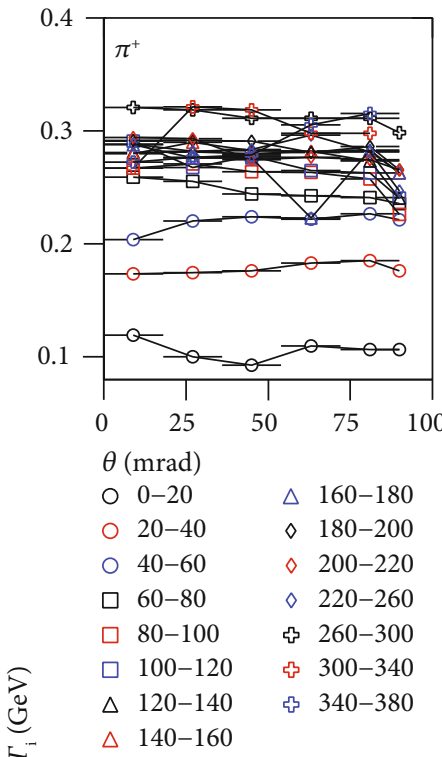

(a)

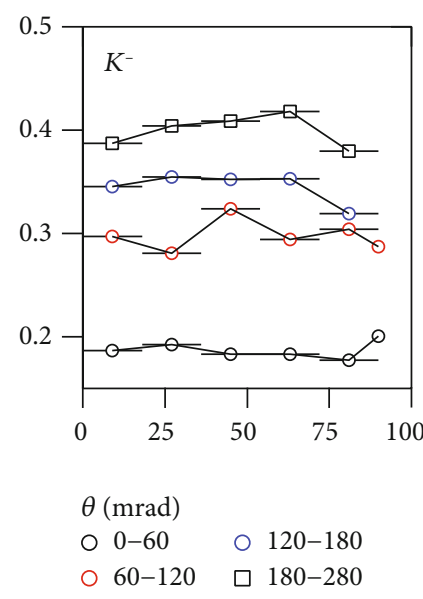

(d)

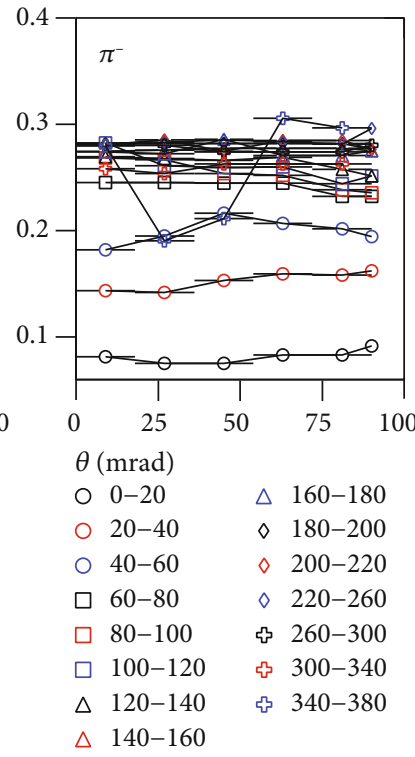

(b)

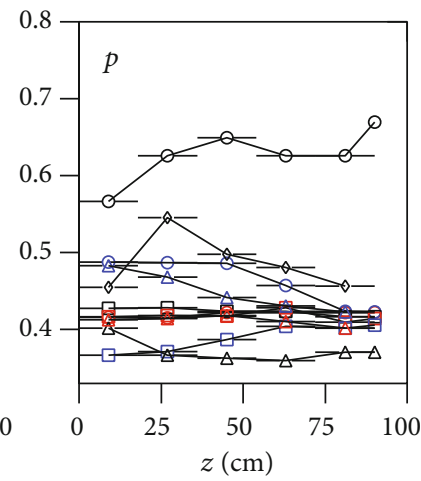

$\theta$ (mrad)
○ 0-20
140-180
○ 20-40
$\triangle 180-220$
○ 40-60
$\triangle 220-260$
60-100
$\triangle 260-300$
ㅁ 100-140

(e)

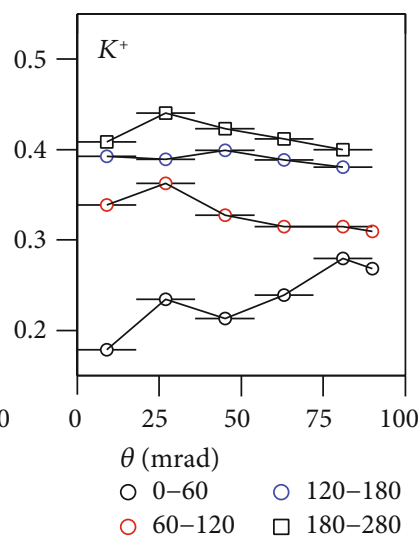

(c)

Figure 15: Same as Figure 14 but showing the dependence of $T_{i}$.

emission angles, $\theta$, and ranges and longitudinal positions, $z$, measured by the NA61/SHINE Collaboration at the SPS

(b) The effective temperature $T$ and rapidity shifts $\Delta y$ from the spectra under given experimental conditions which limit various $\theta$ and $z$ ranges are obtained. For $\pi^{ \pm}$and $K^{ \pm}, T$ and $\Delta y$ decrease slightly with the increase of $\theta$ and do not change obviously with the increase of $z$. The situation for $p$ is more complex due to the effect of leading protons. There is no obvious change in $T$ and $\Delta y$ when $z$ increases due to a not too large energy loss in a not too long graphite target. Both $T$ and $\Delta y$ depend on models. In particular, $T$ contains the contribution of the flow effect, which is not ideal to describe the excitation degree of the emission source (c) The fraction $k(1-k)$ of nonleading (leading) protons in total protons from the spectra in various $\theta$ and $z$ ranges are obtained. There is no obvious change in the dependence of $k(1-k)$ on $\theta$, but some statistical fluctuations. There is a slight increase (decrease) in the dependence of $k(1-k)$ on $z$ with the increase of $z$ due to more energy loss of the beam protons in the target at larger $z$. The effect of leading protons cannot be neglected at the SPS energies. It is expected that $k$ $(1-k)$ will be larger (smaller) at both lower $(\leq$ several $\mathrm{GeV})$ and higher energies ( $\geq$ dozens of $\mathrm{GeV}$ )

(d) The average transverse momentum $\left\langle p_{T}\right\rangle$ and initial quasitemperature $T_{i}$ from the spectra in various $\theta$ and $z$ ranges are obtained. For $\pi^{ \pm}$and $K^{ \pm}$, there are increases in $\left\langle p_{T}\right\rangle$ and $T_{i}$ when $\theta$ increases. The situation for $p$ is complex due to the effect of leading 


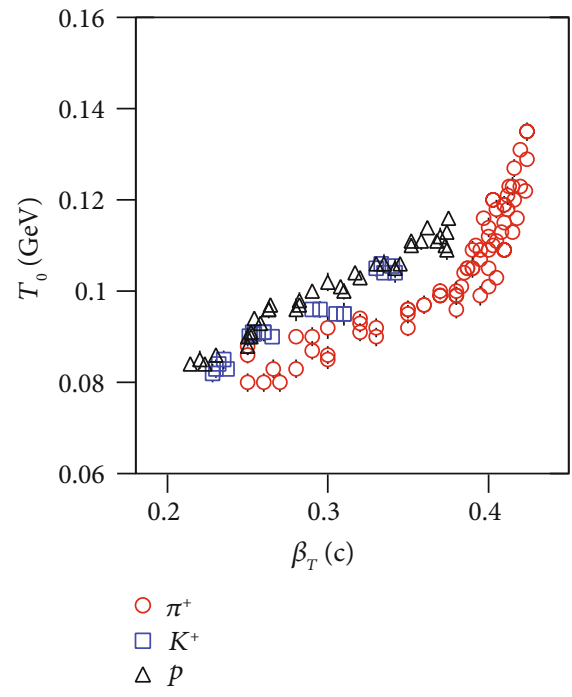

FIgURE 16: Relation between $T_{0}$ and $\beta_{T}$ from the blast-wave model. The symbols represent the results from the spectra of positive particles.

protons. There is no obvious change in $\left\langle p_{T}\right\rangle$ and $T_{i}$ when $z$ increases due to a not too large energy loss in a not too long graphite target. Both $\left\langle p_{T}\right\rangle$ and $T_{i}$ are model dependent due to the fact that they are obtained from the model which fits the data

(e) The behaviors of effective temperature, rapidity shifts, fraction of nonleading (leading) protons, average transverse momentum, and initial quasitemperature obtained from the fits of a multisource thermal model to the NA61/SHINE data can be explained in terms of cascade collisions in the target, stopping power of the target, energy loss of the beam protons in the target, and so on. This paper provides new evidence for the effectiveness of the multisource thermal model, though there is no connection with a possible formation of a Quark-Gluon Plasma as what was being considered was a small system

\section{Data Availability}

The data used to support the findings of this study are included within the article and are cited at relevant places within the text as references.

\section{Ethical Approval}

The authors declare that they are in compliance with ethical standards regarding the content of this paper.

\section{Disclosure}

The funding agencies have no role in the design of the study; in the collection, analysis, or interpretation of the data; in the writing of the manuscript; or in the decision to publish the results.

\section{Conflicts of Interest}

The authors declare that there are no conflicts of interest regarding the publication of this paper.

\section{Acknowledgments}

Communications from Debasish Das are highly acknowledged. Authors P.P.Y., M.Y.D., and F.H.L. acknowledge the financial supports from the National Natural Science Foundation of China under Grant Nos. 11575103 and 11847311, the Shanxi Provincial Innovative Foundation for Graduate Education Grant No. 2019SY053, the Scientific and Technological Innovation Programs of Higher Education Institutions in Shanxi (STIP) under Grant No. 201802017, the Shanxi Provincial Natural Science Foundation under Grant No. 201701D121005, and the Fund for Shanxi "1331 Project" Key Subjects Construction. Author R.S. acknowledges the financial supports from ALICE Project No. SR/MF/PS01/2014-IITI(G) of the Department of Science and Technology (DST), Government of India.

\section{References}

[1] STAR Collaboration, J. Adams, M. M. Aggarwal et al., "Experimental and theoretical challenges in the search for the quarkgluon plasma: the STAR Collaboration's critical assessment of the evidence from RHIC collisions," Nuclear Physics A, vol. 757, no. 1-2, pp. 102-183, 2005.

[2] PHENIX Collaboration, K. Adcox, S. S. Adler et al., "Formation of dense partonic matter in relativistic nucleus-nucleus collisions at RHIC: experimental evaluation by the PHENIX collaboration," Nuclear Physics A, vol. 757, no. 1-2, pp. 184283, 2005.

[3] J. F. Grosse-Oetringhaus and ALICE Collaboration, "Overview of ALICE results at Quark Matter 2014," Nuclear Physics A, vol. 931, pp. 22-31, 2014.

[4] PHENIX Collaboration, S. S. Adler, S. Afanasiev et al., "Systematic studies of the centrality and $\sqrt{s_{N N}}$ dependence of the $d E_{T} / d \eta$ and $d N_{c h} / d \eta$ in heavy ion collisions at midrapidity," Physical Review C, vol. 71, no. 3, article 049901, 2005.

[5] S. M. Troshin and N. E. Tyurin, "Centrality in small systems at the LHC energies and beyond," 2017, http://arxiv.org/abs/ 1711.01069.

[6] STAR Collaboration, J. Adam, L. Adamczyk et al., "Azimuthal harmonics in small and large collision systems at RHIC top energies," Physical Review Letters, vol. 122, no. 17, article 172301, 2019.

[7] J. Jia, M. Zhou, and A. Trzupek, "Revealing long-range multiparticle collectivity in small collision systems via subevent cumulants," Physical Review C, vol. 96, no. 3, article 034906, 2017.

[8] S. Chatterjee, A. K. Dash, and B. Mohanty, "Contrasting freezeouts in large versus small systems," Journal of Physics G: Nuclear and Particle Physics, vol. 44, no. 10, article 105106, 2017.

[9] NA35 Collaboration, T. Alber, H. Appelshäuser et al., "Charged particle production in proton-, deuteron-, oxygenand sulphur-nucleus collisions at $200 \mathrm{GeV}$ per nucleon," The European Physical Journal C - Particles and Fields, vol. 2, no. 4, pp. 643-659, 1998. 
[10] ATLAS Collaboration, M. Aaboud, G. Aad et al., "Measurement of quarkonium production in proton-lead and protonproton collisions at $5.02 \mathrm{TeV}$ with the ATLAS detector," The European Physical Journal C, vol. 78, no. 3, p. 171, 2018.

[11] The LHCb Collaboration, R. Aaij, B. Adeva et al., "Prompt $\Lambda_{c}^{+}$ production in $\mathrm{pPb}$ collisions at $\sqrt{s_{N N}}=5.02 \mathrm{TeV}$," Journal of High Energy Physics, vol. 2019, no. 2, p. 102, 2019.

[12] ALICE Collaboration, S. Acharya, S. Acharya et al., "Energy dependence of exclusive $J / \psi$ photoproduction off protons in ultra-peripheral $\mathrm{p}-\mathrm{Pb}$ collisions at $\sqrt{s_{N N}}=5.02 \mathrm{TeV}$," The European Physical Journal C, vol. 79, no. 5, p. 402, 2019.

[13] CMS Collaboration, A. M. Sirunyan, A. Tumasyan et al., "Measurement of exclusive $\rho(770) 0$ photoproduction in ultrape-ripheral $\mathrm{pPb}$ collisions at $\sqrt{s_{N N}}=5.02 \mathrm{TeV}$," The European Physical Journal C, vol. 79, p. 702, 2019.

[14] PHENIX Collaboration, S. S. Adler, S. Afanasiev et al., "Midrapidity neutral pion production in proton proton collisions at $\sqrt{s}=200 \mathrm{GeV}$," Physical Review Letters, vol. 91, article 241803, 2003.

[15] STAR Collaboration, J. Adams, M. M. Aggarwal et al., "Pion, kaon, proton and anti-proton transverse momentum distributions from $\mathrm{p}+\mathrm{p}$ and $\mathrm{d}+\mathrm{Au}$ collisions at $\sqrt{\mathrm{s}}=200 \mathrm{GeV}$," Physics Letters B, vol. 616, pp. 8-16, 2005.

[16] STAR Collaboration, J. Adams, C. Adler et al., "Cross-sections and transverse single spin asymmetries in forward neutral pion production from proton collisions at $\sqrt{s_{N N}}=200 \mathrm{GeV}$," Physical Review Letters, vol. 92, article 171801, 2004.

[17] Q. H. Xu and for the STAR Collaboration, "Measurements of $\Lambda$ and $\bar{\Lambda}$ polarization in longitudinally polarized protonproton collisions at $\sqrt{s_{N N}}=200 \mathrm{GeV}$ at STAR," AIP Conf. Proc., vol. 842, pp. 71-73, 2006.

[18] P. Staszel and for the BRAHMS collaboration, "The rapidity dependence of the proton-to-pion ratio in $\mathrm{Au}+\mathrm{Au}$ and $\mathrm{p}+\mathrm{p}$ collisions at $\sqrt{s_{N N}}=62.4 \mathrm{GeV}$ and $200 \mathrm{GeV}$," Nuclear Physics $A$, vol. 830, pp. 825c-828c, 2009.

[19] P. Christiansen and for BRAHMS Collaboration, "Rapidity dependence of net protons at $\sqrt{s_{N N}}=200 \mathrm{GeV}$," Nuclear Physics A, vol. 721, pp. 239c-242c, 2003.

[20] PHENIX Collaboration, S. S. Adler, S. Afanasiev et al., "Scaling properties of proton and anti-proton production in $\sqrt{s_{N N}}=$ $200 \mathrm{GeV}$ Au+Au collisions," Physical Review Letters, vol. 91, article 172301, 2003.

[21] G. Renault and for the STAR Collaboration, "Proton-lambda correlations in Au-Au collisions at $\sqrt{s_{N N}}=200 \mathrm{GeV}$ from the STAR experiment," Acta Physica Hungarica, vol. 24, pp. 131-137, 2005.

[22] BRAHMS Collaboration, I. Arsene, I. G. Bearden et al., "Nuclear modification factor for charged pions and protons at forward rapidity in central $\mathrm{Au}+\mathrm{Au}$ collisions at $200 \mathrm{GeV}$," Physics Letters B, vol. 650, pp. 219-223, 2007.

[23] STAR Collaboration, B. I. Abelev, M. M. Aggarwal et al., "Energy dependence of $\pi^{ \pm}, p$ and $\bar{p}$ transverse momentum spectra for $\mathrm{Au}+\mathrm{Au}$ collisions at $\sqrt{s_{N N}}=62.4$ and $200 \mathrm{GeV}$," Physics Letters B, vol. 655, pp. 104-113, 2007.

[24] S. Abreu, S. V. Akkelin, J. Alam et al., "Heavy-ion collisions at the LHC - last call for predictions," Journal of Physics G: Nuclear and Particle Physics, vol. 35, article 054001, 2008.

[25] S. A. Bass, M. Bleicher, W. Cassing et al., "Last call for RHIC predictions," Nuclear Physics A, vol. 661, no. 1-4, pp. 205260, 1999.
[26] J. Cleymans, H. Oeschler, K. Redlich, and S. Wheaton, "Comparison of chemical freeze-out criteria in heavy-ion collisions," Physical Review C, vol. 73, no. 3, article 034905, 2006.

[27] A. Andronic, P. Braun-Munzinger, and J. Stachel, "Hadron production in central nucleus-nucleus collisions at chemical freezeout," Nuclear Physics A, vol. 772, no. 3-4, pp. 167-199, 2006.

[28] A. Andronic, P. Braun-Munzinger, and J. Stachel, "Thermal hadron production in relativistic nuclear collisions," Acta Physica Polonica B, vol. 40, pp. 1005-1012, 2009.

[29] A. Andronic, P. Braun-Munzinger, and J. Stachel, "The horn, the hadron mass spectrum and the QCD phase diagram - the statistical model of hadron production in central nucleusnucleus collisions," Nuclear Physics A, vol. 834, no. 1-4, pp. 237c-240c, 2010.

[30] A. Puglisi, A. Sarracino, and A. Vulpiani, "Temperature in and out of equilibrium: a review of concepts, tools and attempts," Physics Reports, vol. 709-710, pp. 1-60, 2017.

[31] F.-H. Liu, Y.-Q. Gao, T. Tian, and B.-C. Li, "Unified description of transverse momentum spectrums contributed by soft and hard processes in high-energy nuclear collisions," The European Physical Journal A, vol. 50, no. 6, p. 94, 2014.

[32] NA61/SHINE Collaboration, N. Abgrall, A. Aduszkiewicz et al., "Measurements of $\pi^{ \pm}, K^{ \pm}$and proton double differential yields from the surface of the T2K replica target for incoming $31 \mathrm{GeV} / \mathrm{c}$ protons with the NA61/SHINE spectrometer at the CERN SPS," The European Physical Journal C, vol. 79, article $100,2019$.

[33] J. Cleymans and D. Worku, "Relativistic thermodynamics: transverse momentum distributions in high-energy physics," The European Physical Journal A, vol. 48, no. 11, p. 160, 2012.

[34] E. Schnedermann, J. Sollfrank, and U. Heinz, "Thermal phenomenology of hadrons from $200 \mathrm{AGeV}$ S+S collisions," Physical Review C, vol. 48, no. 5, pp. 2462-2475, 1993.

[35] Z.-B. Tang, Y.-C. Xu, L.-J. Ruan, G. van Buren, F.-Q. Wang, and $\mathrm{Z} . \mathrm{B}$. $\mathrm{Xu}$, "Spectra and radial flow in relativistic heavy ion collisions with Tsallis statistics in a blast-wave description," Physical Review C, vol. 79, no. 5, article 051901(R), 2009.

[36] H.-L. Lao, F.-H. Liu, B.-C. Li, and M.-Y. Duan, "Kinetic freezeout temperatures in central and peripheral collisions: which one is larger?," Nuclear Science and Techniques, vol. 29, no. 6 , p. 82, 2018.

[37] H.-L. Lao, F.-H. Liu, B.-C. Li, M.-Y. Duan, and R. A. Lacey, "Examining the model dependence of the determination of kinetic freeze-out temperature and transverse flow velocity in small collision system," Nuclear Science and Techniques, vol. 29, no. 11, p. 164, 2018.

[38] F.-H. Liu, C.-X. Tian, M.-Y. Duan, and B.-C. Li, "Relativistic and quantum revisions of the multisource thermal model in high-energy collisions," Advances in High Energy Physics, vol. 2012, Article ID 287521, 9 pages, 2012.

[39] C. Forbes, M. Evans, N. Hastings, and B. Peacock, Statistical Distributions, Fourth Edition, John Wiley \& Sons, Inc., Hoboken, NJ, USA, 2011.

[40] G.-R. Zhou, Probability Theory and Mathematical Statistics, High Edcudation Press, Beijing, China, 1984.

[41] P.-P. Yang, Q. Wang, and F.-H. Liu, "Mutual derivation between arbitrary distribution forms of momenta and momentum components," International Journal of Theoretical Physics, vol. 58, no. 8, pp. 2603-2618, 2019.

[42] F.-H. Liu, "Particle production in au-au collisions at RHIC energies," Physics Letters B, vol. 583, no. 1-2, pp. 68-72, 2004. 
[43] J. Cleymans, "The physics case for the $\sqrt{s_{N N}} \approx 10 \mathrm{GeV}$ energy region," in Walter Greiner Memorial Volume, pp. 91-96, World Scientific, 2018, http://arxiv.org/abs/1711.02882 (2017).

[44] L. J. Gutay, A. S. Hirsch, C. Pajares, R. P. Scharenberg, and B. K. Srivastava, "De-confinement in small systems: clustering of color sources in high multiplicity $\bar{p} p$ collisions at $\sqrt{s}=1.8$ TeV," International Journal of Modern Physics E, vol. 24, article 1550101, 2015.

[45] A. S. Hirsch, C. Pajares, R. P. Scharenberg, and B. K. Srivastava, "De-confinement in high multiplicity protonproton collisions at LHC energies," 2018, http://arxiv.org/abs/1803.02301.

[46] P. Sahoo, S. De, S. K. Tiwari, and R. Sahoo, "Energy and centrality dependent study of deconfinement phase transition in a color string percolation approach at RHIC energies," The European Physical Journal A, vol. 54, no. 8, article 136, 2018.

[47] The CMS Collaboration, V. Khachatryan, A.. M. Sirunyan et al., "Production of leading charged particles and leading charged-particle jets at small transverse momenta in pp collisions at $\sqrt{s}=8 \mathrm{TeV}$," Physical Review D, vol. 92, article 112001, 2015.

[48] A. Szczurek, N. N. Nikolaev, and J. Speth, "Leading proton spectrum from DIS at HERA," Physics Letters B, vol. 428, no. 3-4, pp. 383-390, 1998.

[49] The ZEUS Collaboration, "Leading proton production in deep inelastic scattering at HERA," Journal of High Energy Physics, vol. 2009, no. 6, p. 74, 2009.

[50] H1 Collaboration, F. D. Aaron, C. Alexa et al., "Measurement of the diffractive deep-inelastic scattering cross section with a leading proton at HERA," The European Physical Journal C, vol. 71, article 1578, 2011.

[51] H1 Collaboration, A. Aktas, V. Andreev et al., "Diffractive deep-inelastic scattering with a leading proton at HERA," The European Physical Journal C, vol. 48, pp. 749-766, 2006.

[52] M. Mieskolainen, "Graniitti: a Monte Carlo event generator for high energy diffraction,” 2019, http://arxiv.org/abs/1910.06300.

[53] D.-M. Wei, F.-L. Shao, J. Song, and Y.-F. Wang, "Centrality, system size and energy dependence of charged particle pseudorapidity distribution," International Journal of Modern Physics A, vol. 23, no. 32, pp. 5217-5227, 2008.

[54] F.-H. Liu, "Unified description of (pseudo)rapidity distributions of final-state particles produced in collisions at relativistic energies," Modern Physics Letters A, vol. 23, no. 5, pp. 337346, 2008.

[55] F.-H. Liu, "Dependence of charged particle pseudorapidity distributions on centrality and energy inp(d)Acollisions at high energies," Physical Review C, vol. 78, no. 1, article 014902, 2008.

[56] E. Cuautle and G. Paic, "Radial flow afterburner for event generators and the baryon puzzle," Journal of Physics G: Nuclear and Particle Physics, vol. 35, no. 7, article 075103, 2008.

[57] STAR Collaboration, B. I. Abelev, M. M. Aggarwal et al., "Systematic measurements of identifled particle spectra in pp, d+au and au+au collisions from STAR," Physical Review C, vol. 79, article 034909, 2009.

[58] W.-J. Xie, "Transverse momentum spectra in high-energy nucleus-nucleus, proton-nucleus and proton-proton collisions," Chinese Physics C, vol. 35, no. 12, pp. 1111-1119, 2011.

[59] D. Thakur, S. Tripathy, P. Garg, R. Sahoo, and J. Cleymans, "Indication of a differential freeze-out in proton-proton and heavy-ion collisions at RHIC and LHC energies," Advances in High Energy Physics, vol. 2016, Article ID 4149352, 13 pages, 2016. 

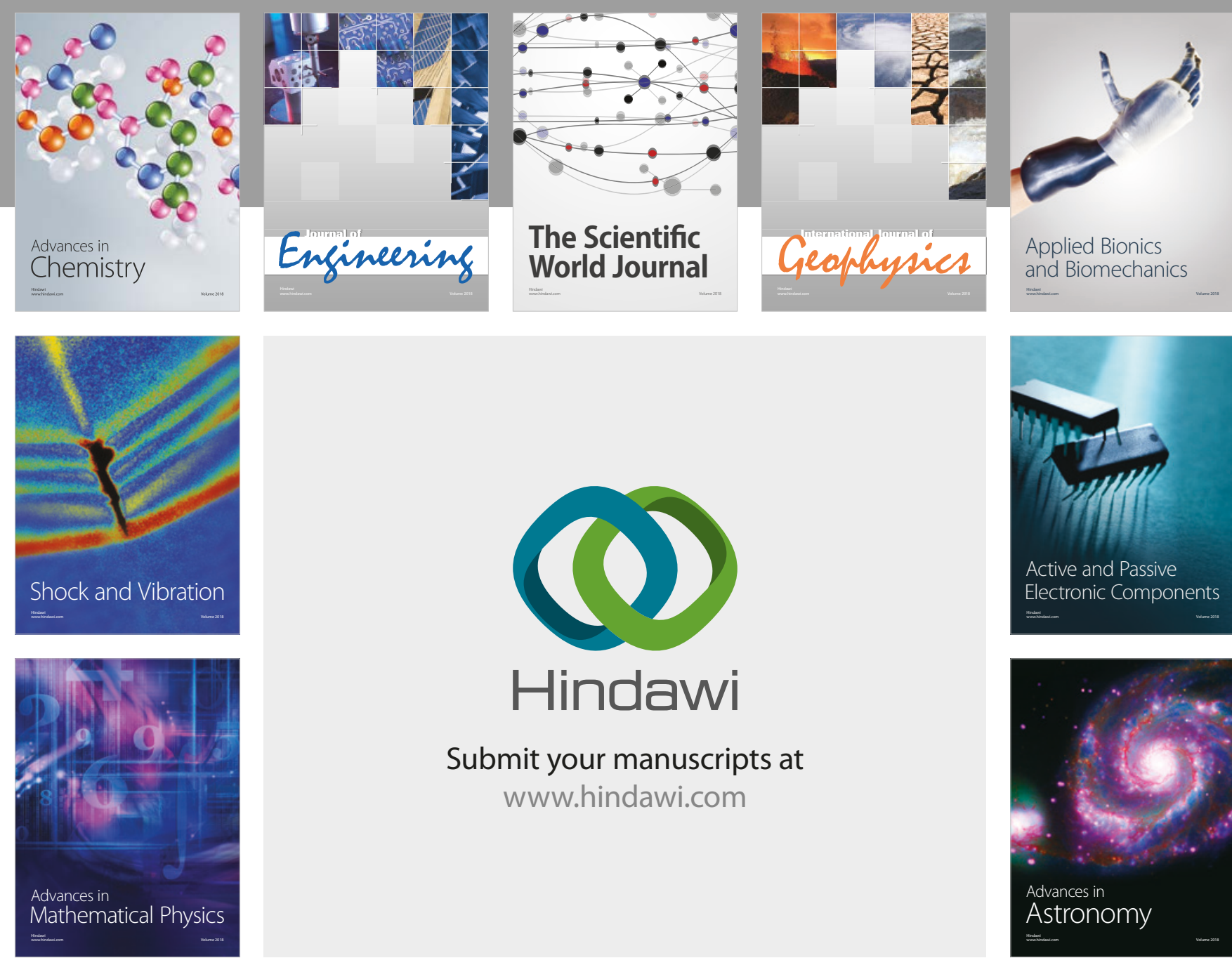

Submit your manuscripts at

www.hindawi.com

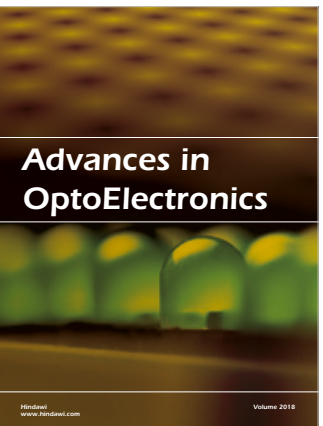

\section{Rotcting Machinery}
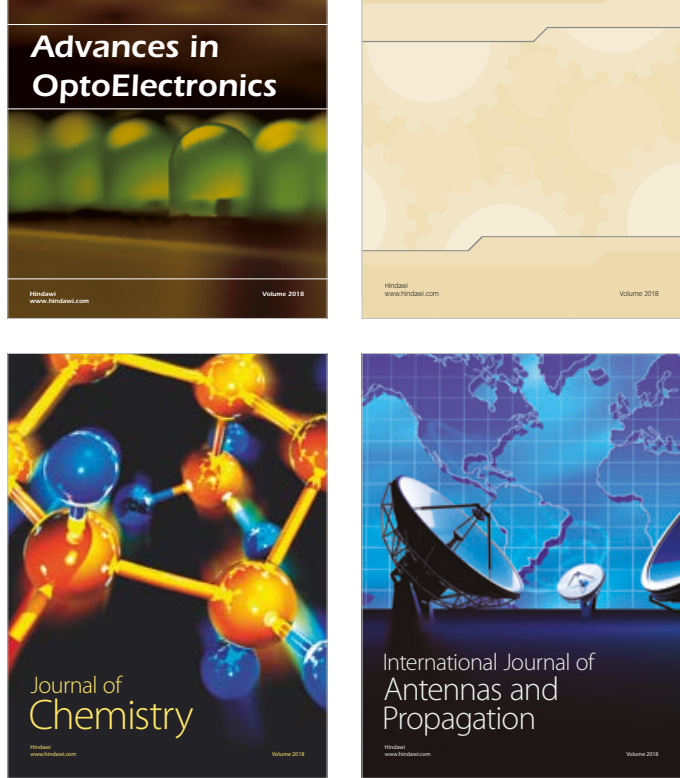

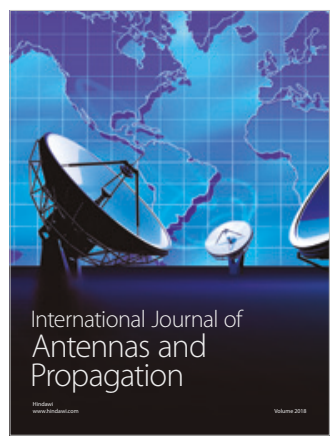

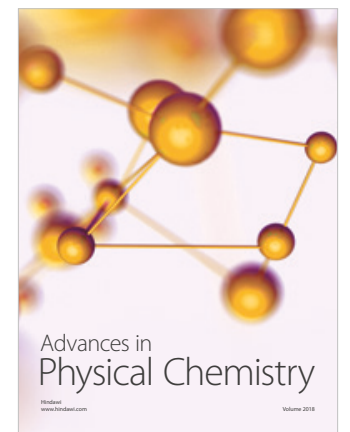

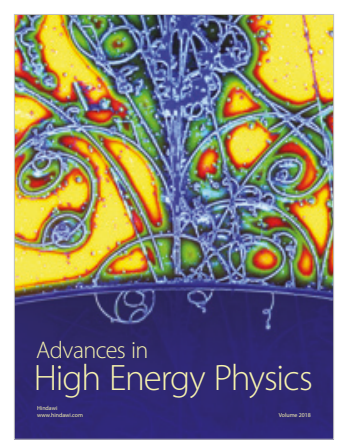

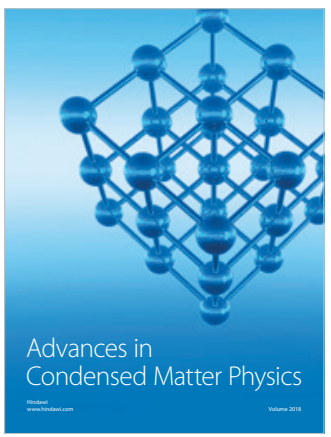

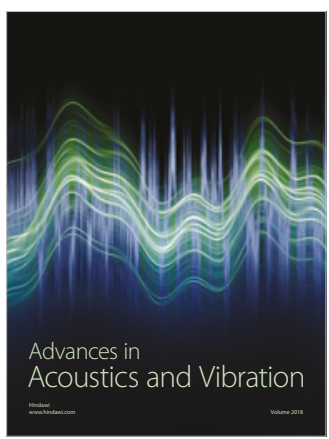

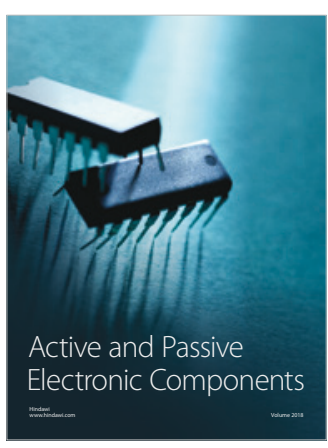
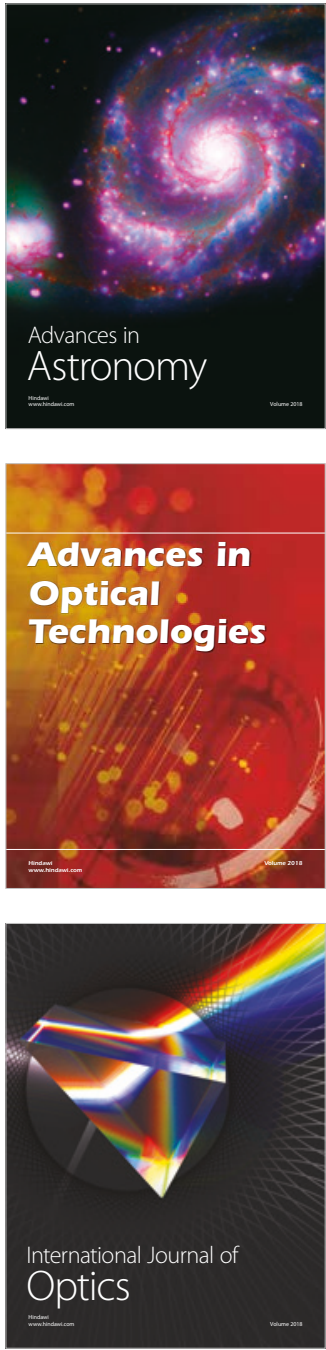